\title{
Xin-Ji-Er-Kang Alleviates Myocardial Infarction-Induced Cardiovascular Remodeling in Rats by Inhibiting Endothelial Dysfunction
}

\author{
Pan Cheng, 1 Feng-zhen Lian $\mathbb{D}^{1},{ }^{1}$ Xiao-yun Wang, ${ }^{1}$ Guo-wei Cai $\mathbb{D}^{1},{ }^{1}$ Guang-yao Huang $\mathbb{D}^{1},{ }^{1}$ \\ Mei-ling Chen, ${ }^{2}$ Ai-zong Shen $\mathbb{D}^{3},{ }^{3}$ and Shan Gao $\mathbb{D}^{1,2}$ \\ ${ }^{1}$ Department of Pharmacology, Basic Medical College, Anhui Medical University, Hefei 230032, China \\ ${ }^{2}$ Cancer Hospital, Chinese Academy of Sciences, Hefei 230032, China \\ ${ }^{3}$ The First Affiliated Hospital of USTC, Division of Life Sciences and Medicine, University of Science and Technology of China, \\ Hefei, Anhui 230001, China
}

Correspondence should be addressed to Ai-zong Shen; ahslyysaz@126.com and Shan Gao; gaoshan@ahmu.edu.cn

Pan Cheng, Feng-zhen Lian, and Xiao-yun Wang contributed equally to this work.

Received 18 February 2019; Revised 29 April 2019; Accepted 26 May 2019; Published 25 June 2019

Academic Editor: Kazim Husain

Copyright (C) 2019 Pan Cheng et al. This is an open access article distributed under the Creative Commons Attribution License, which permits unrestricted use, distribution, and reproduction in any medium, provided the original work is properly cited.

\begin{abstract}
The present study was designed to elucidate the beneficial effects of XJEK on myocardial infarction (MI) in rats, especially through the amelioration of endothelial dysfunction (ED). 136 Sprague-Dawley rats were randomized into 13 groups: control group for 0wk $(n=8)$; sham groups for 2, 4, and 6 weeks (wk); MI groups for 2, 4, and 6 wk; MI+XJEK groups for 2, 4, and 6w k; MI+Fosinopril groups for 2, 4, and $6 \mathrm{wk}(n=8 \sim 10)$. In addition, 8 rats were treated for Evans blue staining and Tetrazolium chloride (TTC) staining to determine the infarct size. Cardiac function, ECG, and cardiac morphological changes were examined. Colorimetric analysis was employed to detect nitric oxide (NO), and enzyme-linked immunosorbent assay (ELISA) was applied to determine N-terminal probrain natriuretic peptide (NT-ProBNP), endothelin-1 (ET-1), angiotensin II (Ang II), asymmetric dimethylarginine (ADMA), tetrahydrobiopterin $\left(\mathrm{BH}_{4}\right)$, and endothelial NO synthase (eNOS) content. The total eNOS and eNOS dimer/(dimer+monomer) ratios in cardiac tissues were detected by Western blot. We found that administration of XJEK markedly ameliorated cardiovascular remodeling (CR), which was manifested by decreased HW/BW ratio, CSA, and less collagen deposition after MI. XJEK administration also improved cardiac function by significant inhibition of the increased hemodynamic parameters in the early stage and by suppression of the decreased hemodynamic parameters later on. XJEK also continuously suppressed the increased NT-ProBNP content in the serum of MI rats. XJEK improved ED with stimulated eNOS activities, as well as upregulated $\mathrm{NO}$ levels, $\mathrm{BH}_{4}$ content, and eNOS dimer/(dimer+monomer) ratio in the cardiac tissues. XJEK downregulated ET-1, Ang II, and ADMA content obviously compared to sham group. In conclusion, XJEK may exert the protective effects on MI rats and could continuously ameliorate $\mathrm{ED}$ and reverse $\mathrm{CR}$ with the progression of $\mathrm{MI}$ over time.
\end{abstract}

\section{Introduction}

Globally, myocardial infarction (MI) has become the leading contributor to the burden of diseases associated with increased risk of heart failure and mortality [1], in spite of the tremendous research efforts over the past years [2]. MI is defined as a pathological event involving ventricular remodeling and myocardial cell necrosis due to significant and sustained ischemia [3]. Being considered as an early response to preserve cardiac function, cardiac hypertrophy can lead to heart failure although the mechanisms involved in the transition are poorly understood [4].

Endothelial dysfunction (ED), characterized by decreased nitric oxide (NO) bioavailability, appears to have a deleterious effect during the long-term process of remodeling [5]. Under physiological conditions, functional endothelial 
NO synthase (eNOS), together with the redox-sensitive cofactor tetrahydrobiopterin $\left(\mathrm{BH}_{4}\right)$, works as a dimeric protein to produce NO, and the eNOS-derived NO serves to promote vascular homeostasis and might affect cardiac myocyte function [6]. However, ventricular remodeling process after MI leads to $\mathrm{BH}_{4}$ oxidation, resulting in the uncoupled eNOSderived superoxide generation, which further augments the remodeling process and deteriorates cardiac function [7]. In addition, endothelin (ET-1), a endothelial-derived vasoconstrictor peptide, maintains the vascular tone in healthy humans. However, its expression and endothelin receptor $\mathrm{A}\left(\mathrm{ET}_{\mathrm{A}}\right)$ levels are upregulated in various cardiovascular disorders like spontaneous hypertension [8], myocardial infarction [9], and atherosclerosis [10]. Evidently, the reninangiotensin system (RAS) is integrally involved in the genesis and progression of various cardiovascular diseases. When RAS is activated, angiotensin II (Ang II) becomes elevated, simultaneously impairing eNOS activity and increasing ET1 levels [11]. Zhou et al. have reported that not only NO bioavailability but also the imbalance between eNOS-derived $\mathrm{NO}$ and ET-1 contributes to ED, ultimately aggravating the MI [12].

Xin-Ji-Er-Kang (XJEK) is a traditional Chinese herbal formula made of fourteen herbal medicines, such as Astragalus mongholicus Bunge, Ophiopogon japonicus (Thunb.) KerGawl, Polygonatum odoratum (Mill.) Druce, Panax ginseng, C.A. Mey., and some other ingredients as well. Numerous clinical and basic researches have revealed the protective effects of XJEK on viral myocarditis [13], MI induced cardiovascular injury [14], and 2-kidney 1-clip (2K1C) induced hypertension $[15,16]$. Our previous studies have shown that the doses of XJEK from 4 to $12 \mathrm{~g} / \mathrm{kg} /$ day, especially $8 \mathrm{~g} / \mathrm{kg} /$ day treatment for 4 weeks, may protect against inflammation, oxidative stress, and MI induced ED in mice [17]. However, it is as yet unclear whether XJEK continues to play a role in MI rats over time or not. This study therefore sets out to assess the effects of XJEK ( $6.2 \mathrm{~g} / \mathrm{kg} / \mathrm{day}$, calculated from dose of mice) on cardiac function abnormalities, cardiovascular remodeling, and $\mathrm{ED}$ over time and attempts to explore the potential mechanisms focusing on ED.

\section{Materials and Methods}

2.1. Animals and Chemicals. All procedures were approved by the Institutional Animal Care and Use Committee of Anhui Medical University. A total of 136 male SpragueDawley rats (220-250 g) were obtained from Shanghai Slac Laboratory Animal Corp. Ltd. (Certificate No.SCXK (HU) 2012-0002) and ventilated with room air. These animals were randomized into the following groups: control group for 0wk $(n=8)$; sham groups for 2,4 , and 6 weeks (wk); MI groups for 2, 4, and $6 \mathrm{wk}$; MI+XJEK groups for 2, 4, and $6 \mathrm{wk}$; MI+Fosinopril groups for 2, 4, and $6 \mathrm{wk}(n$ $=8 \sim 10)$. Another eight rats were treated for Evans blue staining and Tetrazolium chloride (TTC) staining. Animals in XJEK treatment groups received an intragastric gavage with XJEK at $6.2 \mathrm{~g} / \mathrm{kg} / \mathrm{d}$ (calculated from dose of mice); Fosinopril treatment groups were administered $1.5 \mathrm{mg} / \mathrm{kg} / \mathrm{d}$ by intragastric gavage, while those in sham and MI groups were dealt with distilled water. Fosinopril is an angiotensin converting enzyme inhibitor that effectively reduces vascular resistance and improves cardiac output. XJEK was acquired from the Hefei Seven Star Medical Science and Technology Company and Fosinopril was obtained from Bristol-Myers Squibb (Shanghai, China, AAM6233).

\subsection{Establishment of MI Model and Measurement of Infarct} Area at Risk. The MI model was induced by ligation of left anterior descending coronary artery (LAD) and animals undergoing a sham operation were similarly treated, except that the suture around the coronary artery only passed through the muscle without being tied as described previously [12]. Briefly, male rats were anesthetized with sodium pentobarbital $1 \%(50 \mathrm{mg} / \mathrm{kg}$, i.p.) and ventilated with positive pressure via a tube inserted into the trachea and connected to a small animal respirator (BL420S, Chengdu Techman Software Co., Ltd, China). When the adequacy of anesthesia was monitored by observation of slow breathing, loss of muscular tone, and no response to surgical manipulation, a left thoracotomy was performed via the third intercostals space, and the left anterior descending coronary artery was ligated using a 5-0 silk suture. Then, the thoracotomy site was closed. The successful MI model was confirmed not only by real-time ECG monitoring, i.e., a ST segment elevation, but also by visual inspection of LV color alteration. After surgery all animals were injected with cefoxitin sodium (200 $\mathrm{mg} / \mathrm{kg} /$ day) for three consecutive days.

Twenty-four hours after ligation of LAD, heart sections of eight rats were stained with Evans blue/TTC to determine the infarct size as previously described [18]. Evans blue stained areas indicated nonischemia area. Blue, white, and red parts in the heart represented normal myocardium, infarct area, and ischemic area, respectively. White plus red part indicated the area at risk. Photos were captured using a digital camera, and then the relative infarct size could be analyzed with Image $\mathrm{J}(1.61)$.

2.3. Measurement of ECG. During the experimental process, the BL-420 biological function experiment system was used in order to monitor and record the electrocardiogram (ECG) of standard limb lead II as described previously [19]. The height and width of P, T, S wave, QT interval, and P-R interval of baseline, 1 min after MI and 2, 4, and 6 wks after MI, were measured, respectively, using the image analysis software. The ECG changes of each time point were compared among groups.

2.4. Haemodynamic Parameters. At the end of 0, 2, 4, and 6 wks after MI, the haemodynamic parameters were assessed. Animals were anesthetized with sodium pentobarbital $1 \%$ (50 mg/kg, i.p.), respectively; then the right carotid artery was cannulated with a polyethylene catheter connected to a Statham transducer, and the mean carotid artery pressure was measured. After advancing the catheter inserted into the left ventricle along the right coronary artery, the signals were noted down on a four-channel acquisition system (BL420S; Chengdu Techman Software Co. Ltd). The admittance catheters need to be soaked for a while in the heparin 
saline before its insertion into the common carotid artery to prevent clotting. The left ventricular systolic pressure (LVSP), left ventricular end-diastolic pressure (LVEDP), and rate of rise of left ventricular pressure $\left( \pm \mathrm{dp} / \mathrm{dt}_{\max }\right)$ were recorded respectively.

2.5. Collection of Serum, Thoracic Aorta and Cardiac Tissues. After haemodynamic index detection, blood samples were collected for $10 \mathrm{ml}$ from the heart into tubes pretreated with heparin and centrifuged $3500 \mathrm{r} / \mathrm{min}$ for 10 minutes at the temperature of $4^{\circ} \mathrm{C}$; then the supernatant was in storage at $-80^{\circ} \mathrm{C}$ for future analysis as previously described [20]. Cervical exsanguinations were performed on rats, and the heart weight index $(\mathrm{HW} / \mathrm{BW})$ was calculated by dividing HW by BW after the harvest and weighing of hearts. Lastly, being separated into several parts, heart samples were fixed in $10 \%$ neutral buffer formalin for morphological and immunofluorescence detection or stored in liquid nitrogen for further analyses. Thoracic aortas were also evacuated from rats and then cleaned and maintained in neutral $10 \%$ buffered formalin for further morphological detection.

2.6. Measurement of NO and Enzyme-Linked Immunosorbent Assay (ELISA). NO levels were assessed using NO detection Kit (Nanjing Jiancheng Bioengineering Institute, Nanjing, China) following the manufacturer's instructions. ET-1, Ang II, $\mathrm{BH}_{4}, \mathrm{ET}_{\mathrm{A}}$, N-terminal probrain natriuretic peptide (NTProBNP), asymmetric dimethylarginine (ADMA), and eNOS content in serum or cardiac tissues were assessed by ELISA Kit (Jiangsu Zeyu Biological Technology Co., Ltd, Yancheng, China.) according to the manufacturer's instructions.

2.7. Histological and Morphological Analyses of Heart and Thoracic Aorta. Cardiac tissues and thoracic aorta embedded in paraffin were cut into $5 \mu \mathrm{m}$ thick slices and then dewaxed and performed with haematoxylin and eosin (H\&E) or Van Gieson (VG) staining. Afterwards, the myocyte crosssectional area (CSA), thoracic aorta CSA, total aorta (TAA), area of lumen (LA), aorta radius (AR), luminal radius (LR), and media thickness (MT) of aorta were estimated using Image J (1.61) in digitalized microscopic images. Collagen deposition in cardiac tissues was preliminarily evaluated by VG staining. Stained cardiac tissues were photographed at $200 \times$ by microscope. Collagen deposition was then assessed by the mean optical density of red area. Perivascular collagen area (PVCA) and collagen volume fraction (CVF) were observed under optical microscope as previously described by Ahmed et al. [21] and Bai et al. [22].

2.8. Immunofluorescence. The methods of measuring $\mathrm{ET}_{\mathrm{A}}$ by Immunofluorescence had been described previously [23]. Briefly, the hearts were fixed with $10 \%$ neutral buffer formalin, embedded in paraffin and sectioned into $5 \mu \mathrm{m}$ thick slices. After deparaffinization and antigen activation, sections were incubated with 1:200 primary $\mathrm{ET}_{\mathrm{A}}$ monoclonal antibody (Beijing Biosynthesis biotechnology Co., Ltd.) dilution overnight at $4^{\circ} \mathrm{C}$. Goat anti-rabbit IgG antibody labeled with FITC was used as secondary antibodies. Next, sections were sufficiently rinsed in PBS and then incubated with secondary antibody for $60 \mathrm{~min}$. After rinsing in PBS and mounting on slides with mounting medium with DAPI (Sigma-Aldrich), the fluorescent images were captured by inverted fluorescence microscope (Olympus IX71, Japan).

2.9. Western Blotting Analysis. Proteins were extracted from frozen tissue of the cardiac tissues in control ( $0 \mathrm{wk})$, sham, MI, XJEK and Fosinopril (0, 2, 4, and $6 \mathrm{wk})$ groups, respectively. Protein concentration was determined by BCA Protein Assay Kit (Beyotime). LV myocardial homogenates were subjected to sodium dodecyl sulphate-polyacrylamide gel electrophoresis (SDS-PAGE) on $8-10 \%$ polyacrylamide gel, and proteins were electroblotted on the PVDF membranes (Immobilon-P; Millipore, Bedford, MA, USA). After blocking in $5 \%$ nonfat milk solution at $37^{\circ} \mathrm{C}$ for $1 \mathrm{~h}$, the membranes were soaked with the following primary antibodies in TBS$\mathrm{T}$ solutions overnight at $4^{\circ} \mathrm{C}$ : rabbit anti-eNOS antibody (1:1000, Abcam, USA.), rabbit anti-GAPDH antibody (1:1000,

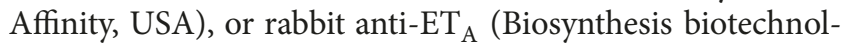
ogy, Beijing.). After incubation with secondary antibodies (goat anti-rabbit IgG, 1:5000; Affinity, USA), the gray value of each band was detected using the super signal enhanced chemiluminescence (ECL; Amersham Biosciences, Little Chalfont, UK) detection system. The relative band intensity was determined using GAPDH as a loading control by ImageJ software. To evaluate the eNOS dimer/ (dimer+monomer) ratio, SDS-resistant eNOS dimers were detected using nondenaturing conditions and low-temperature SDS-PAGE as previously reported and with minor modification [24].

2.10. Statistical Analysis. Results were expressed as mean \pm SEM. Statistical analysis was performed with two-tailed Student's t-test and one-way analysis of variance (ANOVA). Difference was taken statistically significant at $P<0.05$.

\section{Results}

3.1. Survival Rate and Infarct Area 24h after Myocardial Infarction. The survival curve was recorded during the drug treatment period of 2, 4, and 6 wk with XJEK. The data indicated that both XJEK and Fosinopril reduced the mortality of rats with myocardial infarction to a certain extent, but it was not statistically significant (Figures 1(a), 1(b), and 1(c)). As shown in Figure 1(a), white parts in the heart indicated the infarct area, while red represented for ischemic tissue and blue indicated normal myocardium. White plus red part was the area at risk. The result showed that the percentage of infarct area was $37.43 \pm 3.21 \%$ at $24 \mathrm{~h}$ after MI.

3.2. Effects of XJEK on ECG Remodeling in MI Rats. ECG was monitored during the surgical procedures (Figure 2). The $\mathrm{T}$ wave in MI rats group rose obviously after coronary artery ligation compared with that in sham groups. Moreover, the width of P, T, and Q-T interval and the P-R interval increased markedly compared to those in sham groups throughout the 6-week experimental period (2, 4, and $6 \mathrm{wk}, P<0.05$ or $P<0.01)$. However, XJEK (6.2 g/kg) and Fosinopril $(1.5 \mathrm{mg} / \mathrm{kg})$ treatment significantly reduced the height and the width of $\mathrm{P}$ and $\mathrm{T}$ wave and decreased markedly the time of Q-T interval 


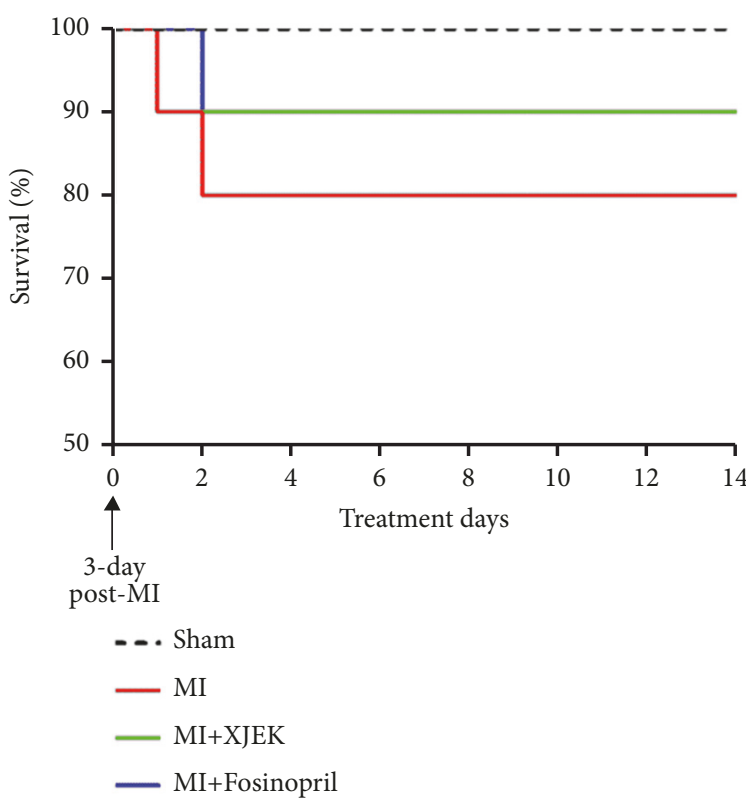

(a)

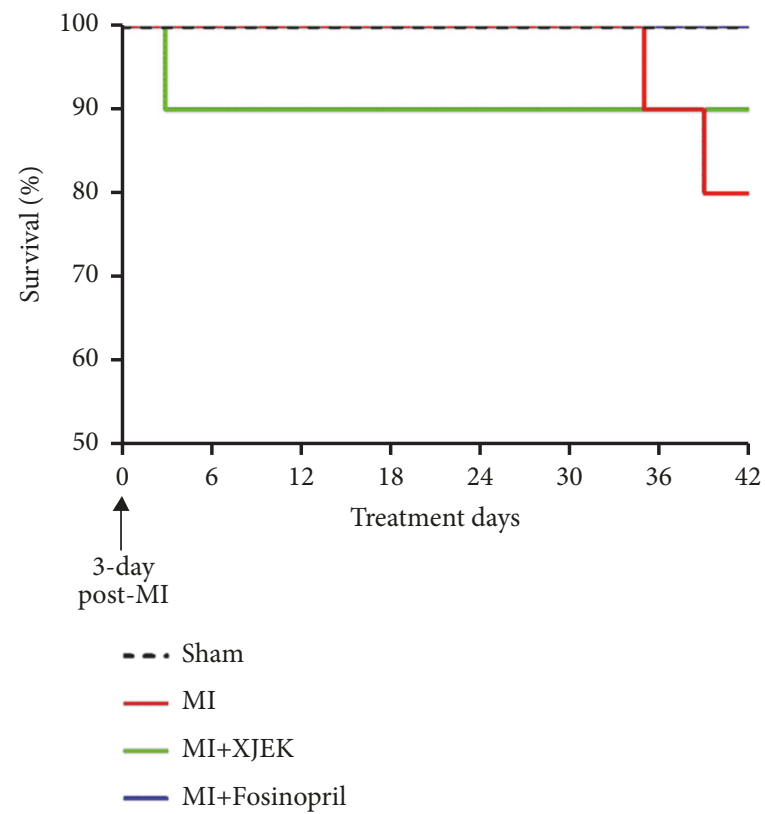

(c)

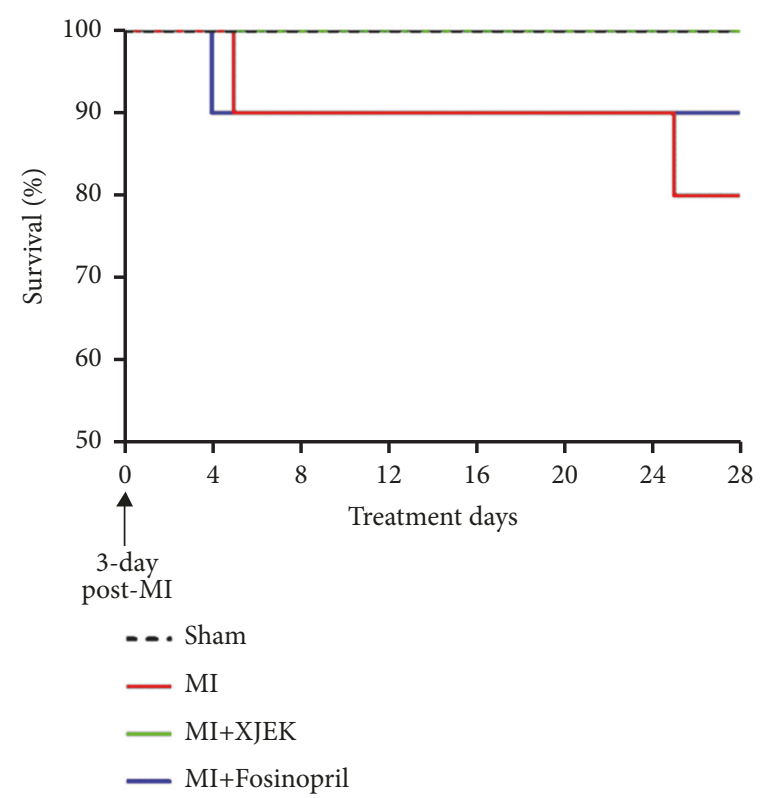

(b)

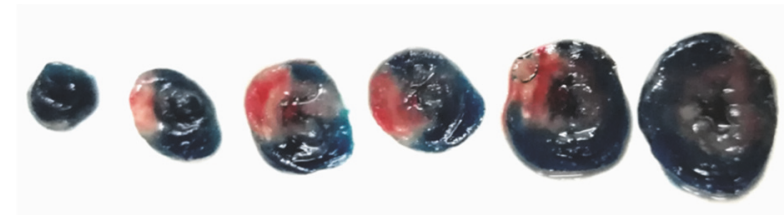

(d)

FIGURE 1: Survival rate and infarct area 24h after myocardial infarction. (a) The survival curve of the 2-week group. (b) The survival curve of the 4-week group. (c) The survival curve of the 6-week group. (d) Representative TTC staining. Infarct size was calculated and quantified by Image J (1.61) ( $n=8)$.

and $\mathrm{P}-\mathrm{R}$ interval compared with those in $\mathrm{MI}$ group $(2,4$, and $6 \mathrm{wk}, P<0.05$ or $P<0.01$; Table 1$)$.

\subsection{Effects of XJEK on Cardiac Function Injury in MI Rats.} We investigated the cardiac function 2, 4, and 6 wk after operation, respectively. There were significant differences in the cardiac function parameters, i.e., HR, ASBP, LVSP, and $\pm \mathrm{dp} / \mathrm{dtmax}$ between sham group and MI group. Compared with sham group, HR was upregulated slightly and there was a significant increase in ASBP, LVSP, and $\pm \mathrm{dp} / \mathrm{dtmax}$ of MI groups for $2 \mathrm{wk}$ and $4 \mathrm{wk}$ ( $2 \mathrm{wk}$ and $4 \mathrm{wk}, P<0.05$ or $P<0.01$ ). In MI group for $6 \mathrm{wk}$; however, LVSP, LVEDP, $+\mathrm{dp} / \mathrm{dtmax}$, and -dp/dtmax were greatly downregulated compared with those of sham group (Table 2). These changes could be blocked by treatment with XJEK for 2, 4, and $6 \mathrm{wk}$, and so could the treatment of Fosinopril.

3.4. Effects of XJEK on NT-ProBNP Content in MI Rats. Serum NT-ProBNP content was significantly higher in the MI rats $(2,4$, and $6 \mathrm{wk}, P<0.01)$. However, its content was 

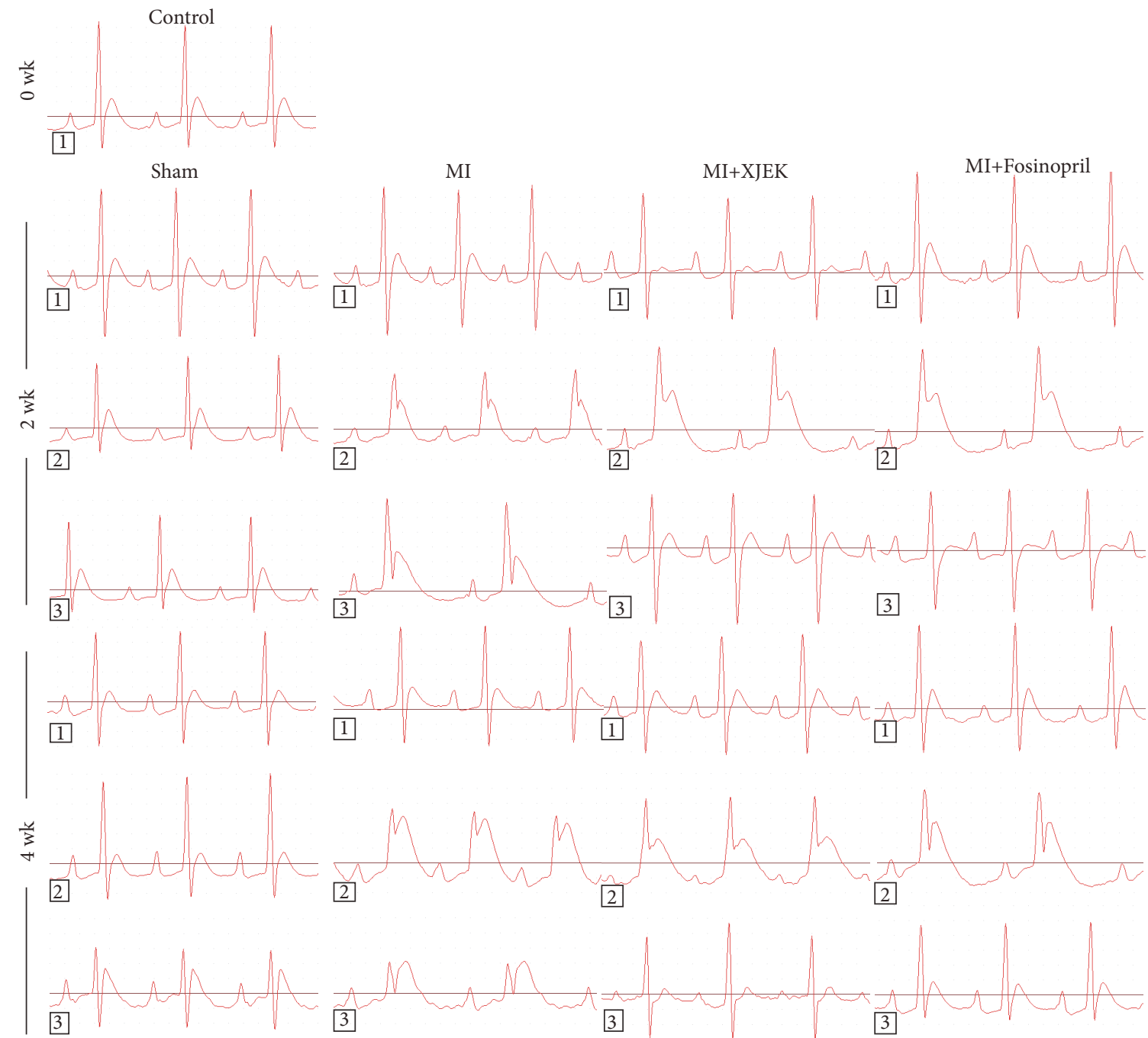

3
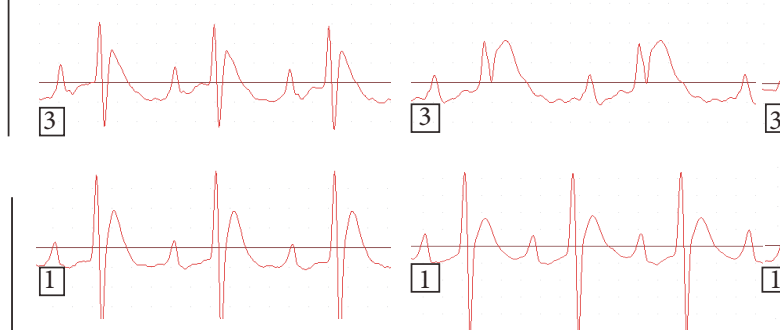

3

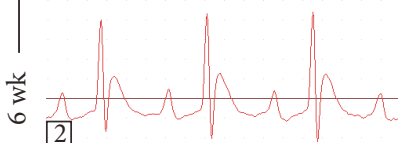

2
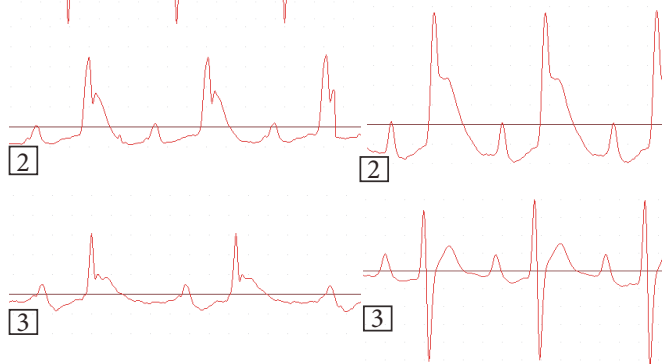

2
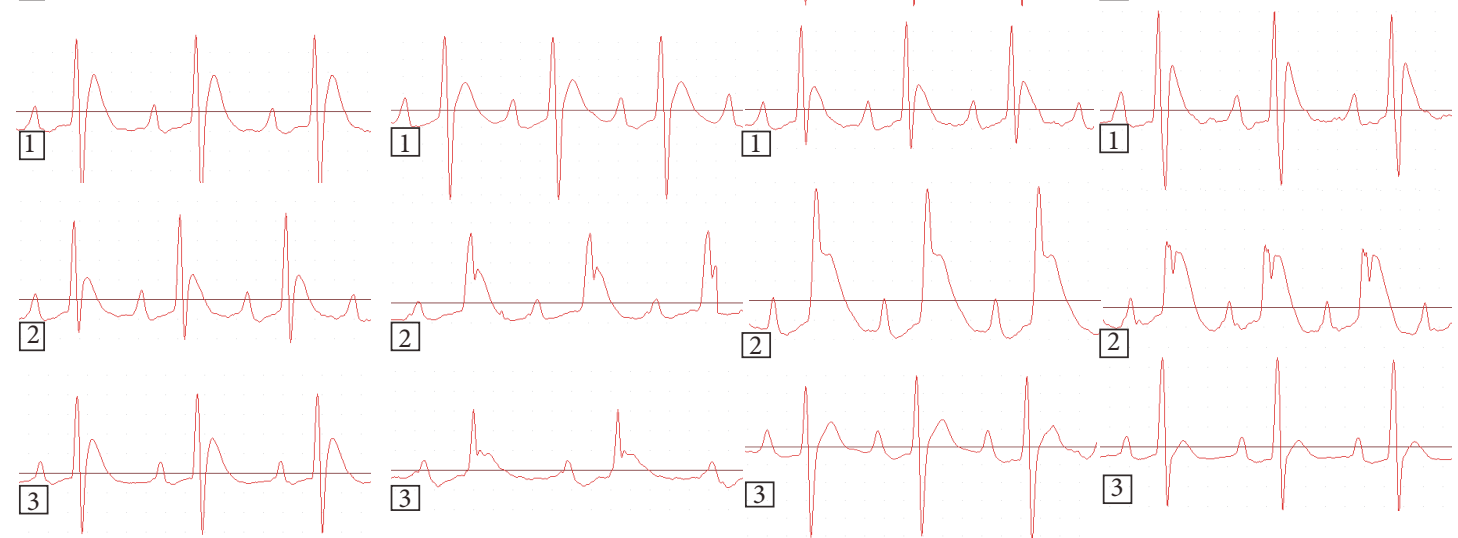

FIGURE 2: Effects of XJEK on ECG remodeling in MI rats. (1) Representative figures of each group on basic (animals were anesthetized but did not receive mechanically ventilated); (2) Representative figures of each group on ischemia 1min (animals were anesthetized and receive mechanically ventilated); (3) Representative figures of each group on ischemia 2, 4, and $6 \mathrm{wk}$ (animals were anesthetized but did not receive mechanically ventilated).

evidently downregulated by XJEK or Fosinopril treatment for 2, 4, and 6 wk, compared with MI groups. As shown in Figure 3(a), the NT-ProBNP content was $770.81 \pm 59.22 \mathrm{pg} \cdot \mathrm{ml}^{-1}$ versus $515.65 \pm 48.57 \mathrm{pg} \cdot \mathrm{ml}^{-1}$ in $2 \mathrm{wk}(P<0.01), 801.39 \pm 56.35$ $\mathrm{pg} \cdot \mathrm{ml}^{-1}$ versus $569.98 \pm 51.88 \mathrm{pg} \cdot \mathrm{ml}^{-1}$ in $4 \mathrm{wk}(P<0.01)$, and
$799.78 \pm 32.06 \mathrm{pg} \cdot \mathrm{ml}^{-1}$ versus $563.31 \pm 17.14 \mathrm{pg} \cdot \mathrm{ml}^{-1}$ in $6 \mathrm{wk}$ $(P<0.001)$ for MI versus XJEK treatments.

Compared with sham group at the same age, NT-ProBNP content in cardiac tissues was significantly higher in the rats with $\mathrm{MI}(2,4$, and $6 \mathrm{wk}, P<0.05)$. However, XJEK 
TABLE 1: Effects of XJEK on ECG remodeling in MI rats.

\begin{tabular}{|c|c|c|c|c|c|c|c|}
\hline Group & Time & $\begin{array}{c}\text { Height of } \\
\mathrm{P}(\mathrm{mv})\end{array}$ & $\begin{array}{l}\text { Width of } \\
\mathrm{P}(\mathrm{ms})\end{array}$ & $\begin{array}{c}\text { Width of } \\
\mathrm{T}(\mathrm{ms})\end{array}$ & $\begin{array}{c}\text { Time of } \\
\mathrm{Q}-\mathrm{T}(\mathrm{ms})\end{array}$ & $\begin{array}{l}\text { Time of } \\
\mathrm{P}-\mathrm{R}(\mathrm{ms})\end{array}$ & $\begin{array}{c}\text { Height of } \\
\mathrm{S}(\mathrm{mv})\end{array}$ \\
\hline Control & 0wk & $0.10 \pm 0.01$ & $19.50 \pm 1.53$ & $27.67 \pm 0.85$ & $52.67 \pm 1.62$ & $46.33 \pm 1.53$ & $-0.33 \pm 0.05$ \\
\hline Sham & \multirow{4}{*}{$2 \mathrm{wk}$} & $0.11 \pm 0.01$ & $20.22 \pm 0.62$ & $27.56 \pm 1.47$ & $50.33 \pm 1.33$ & $45.33 \pm 1.22$ & $-0.23 \pm 0.02$ \\
\hline MI & & $0.10 \pm 0.01^{* *}$ & $20.88 \pm 0.93$ & $39.25 \pm 1.50^{* * *}$ & $60.88 \pm 1.44^{* * *}$ & $53.00 \pm 2.85^{*}$ & $-0.15 \pm 0.03^{*}$ \\
\hline MI+XJEK & & $0.12 \pm 0.01^{\#}$ & $17.25 \pm 0.80$ & $21.13 \pm 2.58^{\#}$ & $40.63 \pm 3.76$ & $45.43 \pm 1.54^{\#}$ & $-0.25 \pm 0.03^{\#}$ \\
\hline MI+Fosinopril & & $0.12 \pm 0.02^{\# \#}$ & $18.88 \pm 2.17^{\#}$ & $20.63 \pm 8.73^{\# \# \#}$ & $20.14 \pm 2.41^{\# \#}$ & $48.38 \pm 5.63^{\#}$ & $-0.25 \pm 0.08^{\#}$ \\
\hline Sham & \multirow{4}{*}{$4 \mathrm{wk}$} & $0.11 \pm 0.01$ & $17.45 \pm 1.06$ & $29.22 \pm 1.61$ & $53.56 \pm 1.97$ & $44.45 \pm 1.47$ & $-0.43 \pm 0.07$ \\
\hline MI & & $0.08 \pm 0.01^{*}$ & $26.40 \pm 1.45^{* * *}$ & $37.38 \pm 3.96$ & $60.22 \pm 3.70$ & $55.40 \pm 0.82^{* * *}$ & $-0.16 \pm 0.03^{* *}$ \\
\hline MI+XJEK & & $0.13 \pm 0.01^{\# \#}$ & $18.09 \pm 0.97^{\# \#}$ & $22.30 \pm 2.37^{\#}$ & $51.00 \pm 3.54$ & $45.73 \pm 2.14^{\# \#}$ & $-0.34 \pm 0.06^{\# \#}$ \\
\hline MI+Fosinopril & & $0.10 \pm 0.01$ & $19.18 \pm 1.13^{\# \#}$ & $26.50 \pm 2.85^{\#}$ & $53.20 \pm 2.51$ & $51.78 \pm 1.53^{\#}$ & $-0.28 \pm 0.02^{\# \#}$ \\
\hline Sham & \multirow{4}{*}{$6 w k$} & $0.10 \pm 0.01$ & $15.90 \pm 1.18$ & $27.89 \pm 1.21$ & $48.44 \pm 1.32$ & $47.44 \pm 1.25$ & $-0.36 \pm 0.04$ \\
\hline MI & & $0.07 \pm 0.01^{*}$ & $22.44 \pm 1.17^{* *}$ & $38.50 \pm 2.82^{* *}$ & $59.63 \pm 3.30^{* *}$ & $51.22 \pm 2.47$ & $-0.15 \pm 0.04^{* *}$ \\
\hline MI+XJEK & & $0.10 \pm 0.01^{\#}$ & $19.33 \pm 0.72^{\#}$ & $29.50 \pm 2.69^{\#}$ & $51.60 \pm 3.34$ & $45.10 \pm 1.62^{\#}$ & $-0.31 \pm 0.04^{\#}$ \\
\hline MI+Fosinopril & & $0.10 \pm 0.01^{\# \#}$ & $18.55 \pm 0.65^{\# \#}$ & $27.45 \pm 2.71^{\#}$ & $50.40 \pm 2.46^{\#}$ & 45. $20 \pm 1.31^{\#}$ & $-0.30 \pm 0.04^{\#}$ \\
\hline
\end{tabular}

Data are represented as mean $\pm \operatorname{SEM}(n=8 \sim 10) .{ }^{*} P<0.05,{ }^{* *} P<0.01$, and ${ }^{* * *} P<0.001$ versus sham group; ${ }^{\#} P<0.05,{ }^{\# \#} P<0.01$, and ${ }^{\# \# \#} P<0.001$ versus MI group.

TABLE 2: Effects of XJEK on cardiac function injury in MI rats.

\begin{tabular}{|c|c|c|c|c|c|c|c|}
\hline Group & Time & $\begin{array}{c}\text { HR } \\
\text { (Times/min) }\end{array}$ & $\begin{array}{c}\text { ASBP } \\
(\mathrm{mmHg})\end{array}$ & $\begin{array}{l}\text { LVEDP } \\
(\mathrm{mmHg})\end{array}$ & $\begin{array}{c}\text { LVSP } \\
(\mathrm{mmHg})\end{array}$ & $\begin{array}{l}+\mathrm{dp} / \mathrm{dtmax} \\
(\mathrm{mmHg} / \mathrm{s})\end{array}$ & $\begin{array}{l}\text { dp/dtmax } \\
(\mathrm{mmHg} / \mathrm{s}) \\
\end{array}$ \\
\hline Control & 0wk & $381.63 \pm 33.58$ & $96.67 \pm 2.92$ & $-16.42 \pm 3.88$ & $109.51 \pm 2.83$ & $4049.67 \pm 237.23$ & $-3317.31 \pm 183.80$ \\
\hline Sham & \multirow{4}{*}{$2 w k$} & $403.14 \pm 38.88$ & $95.31 \pm 3.76$ & $-23.47 \pm 3.79$ & $119.82 \pm 3.06$ & $3972.84 \pm 122.19$ & $-3546.74 \pm 220.85$ \\
\hline MI & & $450.00 \pm 32.07$ & $109.80 \pm 4.06^{*}$ & $-8.92 \pm 4.57^{*}$ & $126.38 \pm 4.20$ & $4572.79 \pm 227.81^{*}$ & $-4031.96 \pm 183.64$ \\
\hline MI+XJEK & & $376.86 \pm 42.73$ & $92.97 \pm 3.94^{\# \#}$ & $-15.67 \pm 8.09$ & $109.07 \pm 4.78^{\#}$ & $3687.44 \pm 228.53^{\#}$ & $-3302.12 \pm 262.12^{\#}$ \\
\hline MI+Fosinopril & & $400.38 \pm 23.00$ & $93.83 \pm 3.86^{\#}$ & $-14.69 \pm 2.89$ & $102.89 \pm 5.34^{\# \#}$ & $3246.49 \pm 198.24^{\# \# \#}$ & $-3012.45 \pm 329.80^{\#}$ \\
\hline Sham & \multirow{4}{*}{$4 \mathrm{wk}$} & $372.64 \pm 12.31$ & $91.64 \pm 1.64$ & $-27.43 \pm 1.79$ & $113.58 \pm 3.46$ & $3749.03 \pm 212.07$ & $-3390.87 \pm 201.06$ \\
\hline MI & & $428.00 \pm 50.08$ & $110.92 \pm 4.63^{* *}$ & $-9.21 \pm 4.25^{* * *}$ & $135.18 \pm 4.73^{* *}$ & $4541.46 \pm 308.14^{*}$ & $-4292.16 \pm 757.17^{*}$ \\
\hline MI+XJEK & & $399.50 \pm 18.09$ & $90.34 \pm 2.57^{\# \# \#}$ & $-20.65 \pm 1.90$ & $107.83 \pm 3.67^{\# \#}$ & $3402.62 \pm 130.43^{\# \#}$ & $-2983.99 \pm 139.16^{\# \#}$ \\
\hline MI+Fosinopril & & $386.40 \pm 41.21$ & $92.41 \pm 2.85^{\# \#}$ & $-20.53 \pm 1.37^{\#}$ & $106.28 \pm 11.90^{\# \#}$ & $3402.17 \pm 235.64^{\# \#}$ & $-3305.10 \pm 383.84^{\# \#}$ \\
\hline Sham & \multirow{4}{*}{$6 \mathrm{wk}$} & $387.30 \pm 18.66$ & $103.51 \pm 4.83$ & $-28.18 \pm 2.69$ & $119.76 \pm 7.11$ & $4279.16 \pm 402.39$ & $-3694.09 \pm 289.27$ \\
\hline MI & & $402.50 \pm 17.21$ & $90.91 \pm 4.48$ & $-7.26 \pm 7.39$ & $107.51 \pm 6.02$ & $3445.35 \pm 416.49$ & $-2870.24 \pm 244.74^{* *}$ \\
\hline MI+XJEK & & $389.33 \pm 10.04$ & $113.42 \pm 4.32^{\# \#}$ & $-22.98 \pm 1.98^{\#}$ & $129.06 \pm 4.97^{\#}$ & $4876.77 \pm 369.09^{\#}$ & $-4270.13 \pm 210.55^{\# \#}$ \\
\hline MI+Fosinopril & & $385.82 \pm 28.82$ & $115.23 \pm 2.72^{\# \#}$ & $-23.84 \pm 1.77^{\#}$ & $128.82 \pm 11.65^{\# \#}$ & $4937.62 \pm 203.45^{\# \#}$ & $-4117.43 \pm 228.77^{\# \#}$ \\
\hline
\end{tabular}

and Fosinopril treatments could normalize the NT-ProBNP content (Figure 3(b)).

3.5. Effects of XJEK on Cardiac Hypertrophy and Cardiac Collagen Deposition in MI Rats. Morphological hypertrophy of heart was featured by apparently increased HW/BW ratio in MI groups ( $2 \mathrm{wk}$ and $4 \mathrm{wk}, P<0.01 ; 6 \mathrm{wk}, P<0.05$; Table 3 and Figure 4). Similarly, as indicated by hematoxylin-eosin (HE) staining of cardiac tissues from MI groups for 2, 4, and $6 \mathrm{wk}$, myocyte CSA, and longitudinal diameter increased markedly compared to those of sham groups $(2,4$, and 6 wk, $P<0.01$; Figures 5(a), 5(b), and 5(c)). However, both the elevated HW/BW and myocyte CSA could be restored by XJEK or Fosinopril treatments for 2, 4, and $6 \mathrm{wk}(2,4$, and $6 \mathrm{wk}, P<0.01$ or $P<0.05)$.
The effects of XJEK treatment for 2, 4, and $6 \mathrm{wk}$ on CVF and PVCA in rat hearts were examined by VG staining. Compared with sham groups, the $\operatorname{CVF}(2,4$, and 6 wk $P<0.01$; Figures 6(a) and 6(c)) and PVCA (2, 4, and 6 wk $P<0.01$; Figures 6(b) and 6(d)) were significantly elevated in MI groups for 2, 4, and $6 \mathrm{wk}$, but evidently reduced in XJEK (2, 4 , and 6 wk, $P<0.01)$ and Fosinopril $(2,4$, and 6 wk, $P<0.01)$ treatments for 2,4 , and $6 \mathrm{wk}$.

3.6. Effects of XJEK on Aortic Remodeling in MI Rats. The effects of XJEK treatment for 2, 4, and 6 wk on the vascular remodeling of the upper thoracic aorta was detected, respectively. Compared with sham group, MI groups for 2, 4, and 6 wk had an increasing trend on TAA, CSA, AR, and Media of the aorta $(2,4$, and $6 \mathrm{wk}, P<0.05$ or $P<0.01$; Figure 7 and 


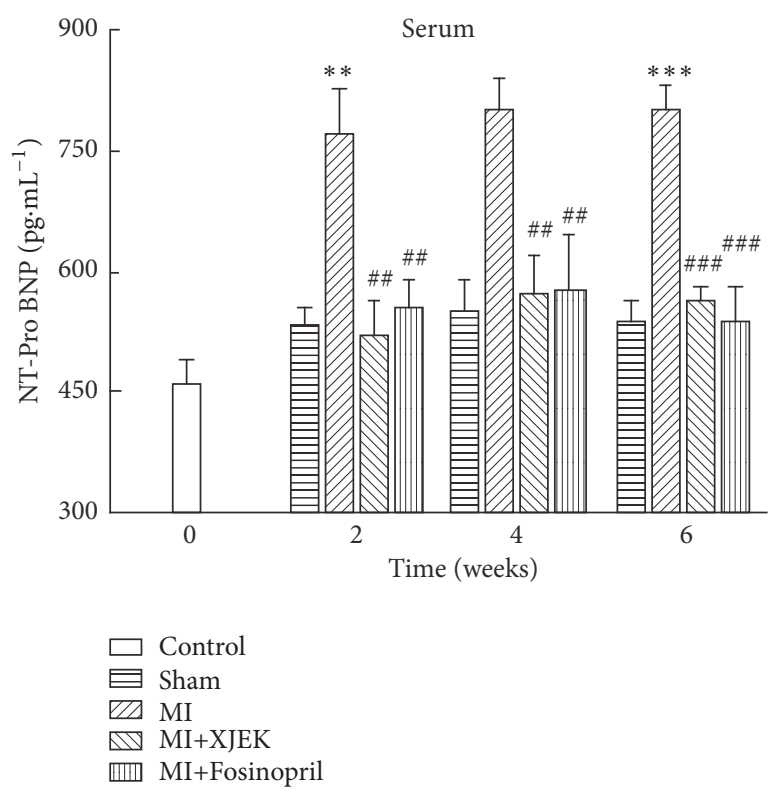

(a)

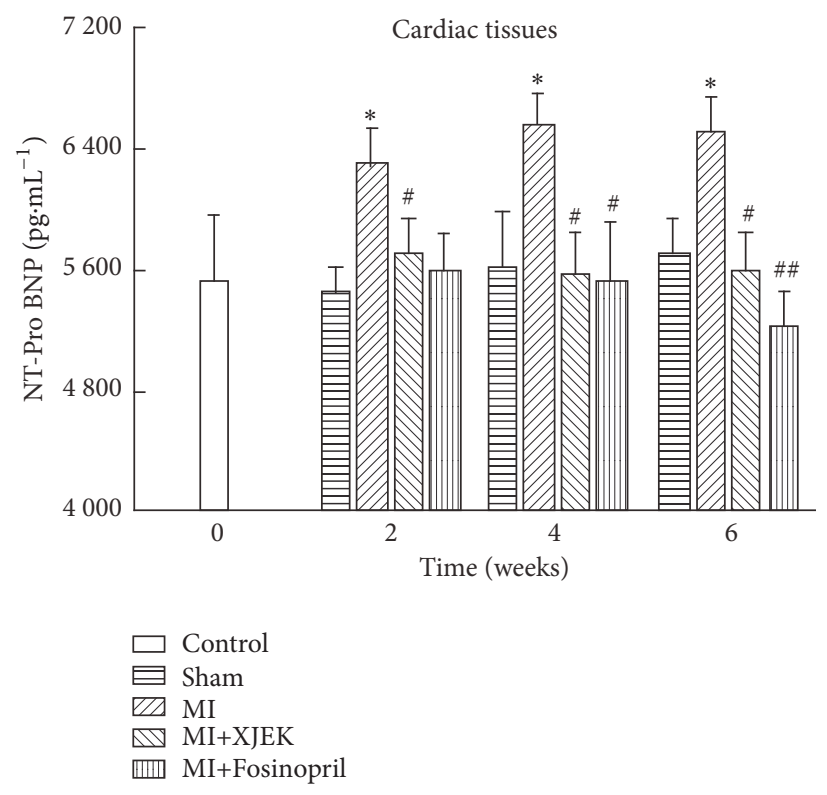

(b)

FIGURE 3: Effects of XJEK on NT-ProBNP content in MI rats. (a) NT-ProBNP content in serum; (b) NT-ProBNP content in cardiac tissues. Data are represented as mean $\pm \operatorname{SEM}(n=8 \sim 10) .{ }^{*} P<0.05,{ }^{* *} P<0.01$, and ${ }^{* * *} P<0.001$ versus sham group; ${ }^{\#} P<0.05,{ }^{\# \#} P<0.01$, and ${ }^{\# \# \#} P<0.001$ versus MI group.

TABLE 3: Effects of XJEK on BW, HW, and HW/BW in MI rats.

\begin{tabular}{lcccc}
\hline Group & Time & BW $(\mathrm{g})$ & HW $(\mathrm{g})$ & HW/BW \\
\hline Control & 0wk & $268.5 \pm 4.31$ & $0.78 \pm 0.03$ & $2.92 \pm 0.13$ \\
\hline Sham & & $339.29 \pm 7.74$ & $0.97 \pm 0.05$ & $2.77 \pm 0.09$ \\
MI & $2 w k$ & $330.40 \pm 9.84$ & $1.07 \pm 0.05$ & $3.24 \pm 0.11^{* *}$ \\
MI+XJEK & & $337.38 \pm 7.49$ & $0.99 \pm 0.04$ & $2.96 \pm 0.06^{\#}$ \\
MI+Fosinopril & & $328.50 \pm 4.41$ & $0.95 \pm 0.02^{\#}$ & $2.94 \pm 0.07^{\#}$ \\
\hline Sham & $4 w k$ & $392.00 \pm 12.50$ & $2.61 \pm 0.11$ \\
MI & & $374.67 \pm 13.07$ & $1.01 \pm 0.03$ & $2.85 \pm 0.07^{* *}$ \\
MI+XJEK & & $380.25 \pm 8.11$ & $2.07 \pm 0.05$ & $2.58 \pm 0.10^{\#}$ \\
MI+Fosinopril & $377.00 \pm 9.94$ & $0.98 \pm 0.03^{\#}$ & $2.56 \pm 0.06^{\# \#}$ \\
\hline Sham & & $419.78 \pm 17.96$ & $0.97 \pm 0.02^{\#}$ & $2.47 \pm 0.11$ \\
MI & $6 w k$ & $396.86 \pm 16.29$ & $0.99 \pm 0.03$ & $2.79 \pm 0.04^{*}$ \\
MI+XJEK & & $407.67 \pm 9.41$ & $1.11 \pm 0.05^{*}$ & $2.57 \pm 0.07^{\#}$ \\
MI+Fosinopril & & $412.45 \pm 10.60$ & $1.00 \pm 0.02^{\#}$ & $2.38 \pm 0.06^{\# \#}$ \\
\hline
\end{tabular}

BW: body weight; HW: heart weight; HW/BW: heart weight index. Data are represented as mean \pm SEM $(n=8 \sim 10) .{ }^{*} P<0.05$ and ${ }^{* *} P<0.01$ versus sham group; ${ }^{\#} P<0.05$ and ${ }^{\# \#} P<0.01$ versus MI group.

Table 4), while XJEK treatment for 2, 4, and $6 \mathrm{wk}$, the TAA, CSA, AR, and Media significantly decreased (2 wk, $4 \mathrm{wk}$, and $6 \mathrm{wk}, P<0.05$ or $P<0.01$; Figure 6 and Table 3$)$. Fosinopril treatment for 2, 4, and 6 wk achieved similar effects $(2,4$, and $6 \mathrm{wk}, P<0.05$ or $P<0.01$; Figure 7 and Table 3 ).

3.7. Effects of XJEK on $\mathrm{BH}_{4}, \mathrm{NO}$, and ADMA Content in $\mathrm{MI}$ Rats. $\mathrm{BH}_{4}$ content in serum and cardiac tissues of rats in $\mathrm{MI}$ groups for 2, 4, and $6 \mathrm{wk}$ was examined by ELISA. $\mathrm{BH}_{4}$ was highly expressed in serum and cardiac tissues of rats in sham groups, while its content decreased in MI groups for 2, 4, and
6 wk (serum, 4 wk, $P<0.05$, and 6 wk, $P<0.01$, Figure 8 (a); cardiac tissues, $4 \mathrm{wk}$ and $6 \mathrm{wk}, P<0.05$, Figure $8(\mathrm{~b}))$. However, its content was evidently upregulated by XJEK or Fosinopril treatment for 2, 4, and $6 \mathrm{wk}$, compared with MI groups.

NO content in serum of MI groups for 2, 4, and $6 \mathrm{wk}$ was significantly decreased and deteriorated over time, compared with sham groups ( $2 \mathrm{wk}, P<0.01,4 \mathrm{wk}$ and $6 \mathrm{wk}, P<0.05)$. But both XJEK and Fosinopril treatment for 2, 4, and 6 wk could prevent NO reduction (Figure $8(\mathrm{c})$ ).

We further examined the influence of XJEK treatment on ADMA levels in serum by ELISA. Compared with sham 

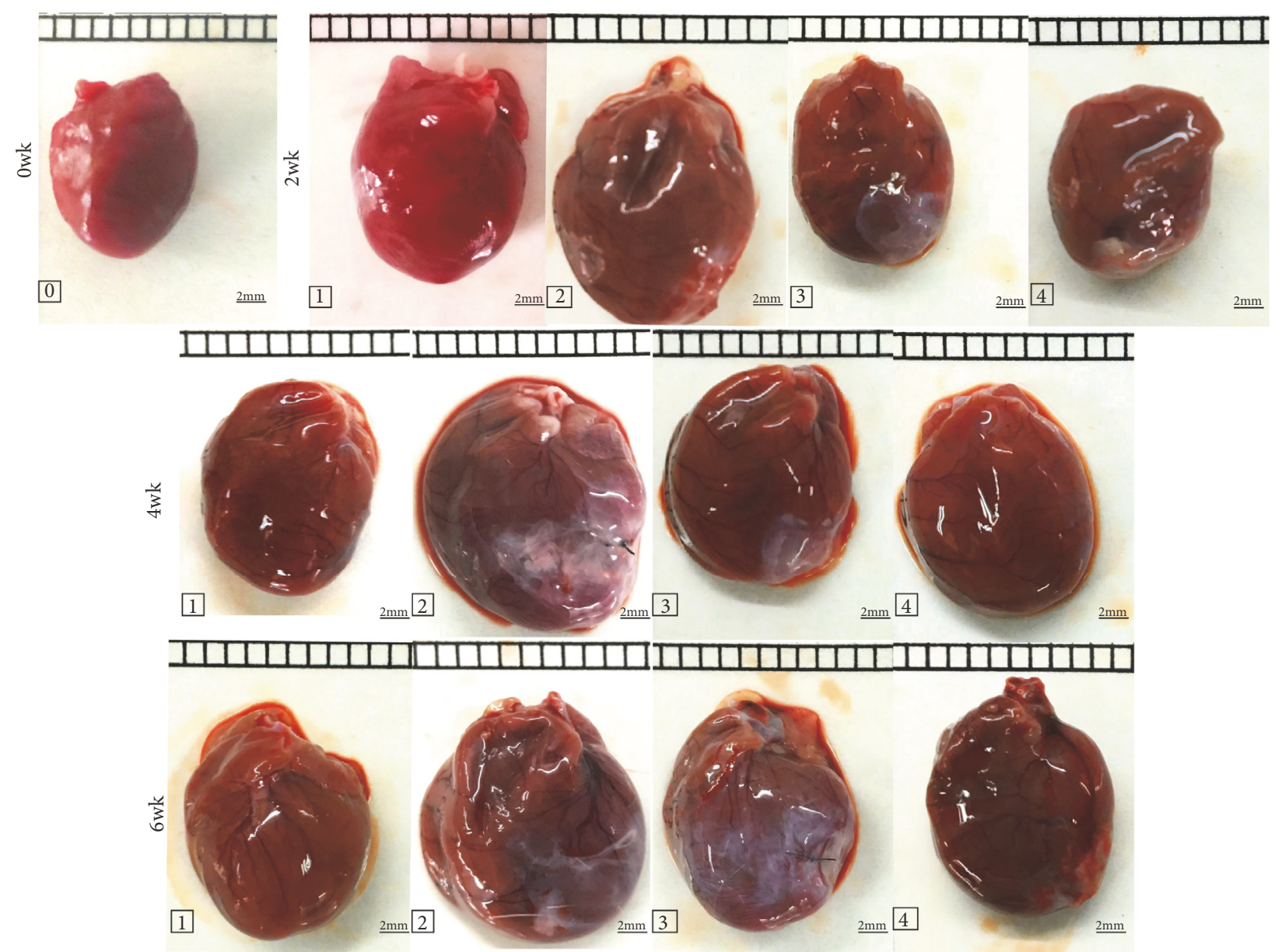

FIgURE 4: Effects of XJEK on HW/BW in MI rats. (0) Control group; (1) sham group; (2) MI group; (3) MI+XJEK group; (4) MI+Fosinopril group.

TABLE 4: Effects of XJEK on thoracic aorta remodeling in MI rats.

\begin{tabular}{lccccccc}
\hline Group & Time & TAA $\left(10^{3} \mathrm{um}^{2}\right)$ & $\mathrm{LA}\left(10^{3} \mathrm{um}^{2}\right)$ & $\mathrm{CSA}\left(10^{3} \mathrm{um}^{2}\right)$ & $\mathrm{AR}(\mathrm{um})$ & $\mathrm{LR}(\mathrm{um})$ & $\mathrm{MT}(\mathrm{um})$ \\
\hline Control & 0wk & $376.57 \pm 11.65$ & $285.52 \pm 10.55$ & $91.06 \pm 4.23$ & $345.97 \pm 5.40$ & $301.15 \pm 5.62$ & $44.81 \pm 2.01$ \\
\hline Sham & & $396.45 \pm 14.85$ & $308.04 \pm 13.86$ & $88.41 \pm 7.81$ & $354.78 \pm 6.73$ & $312.56 \pm 7.11$ & $42.23 \pm 1.27$ \\
MI & $2 \mathrm{wk}$ & $460.73 \pm 24.59^{*}$ & $334.60 \pm 17.95$ & $126.13 \pm 7.64^{* *}$ & $381.95 \pm 10.47^{*}$ & $325.49 \pm 8.99$ & $56.47 \pm 2.21^{* *}$ \\
MI+XJEK & & $424.75 \pm 20.05^{\#}$ & $323.49 \pm 17.03^{\#}$ & $101.26 \pm 4.21^{\# \# \#}$ & $366.73 \pm 8.70^{\#}$ & $319.84 \pm 8.45$ & $46.89 \pm 1.33^{\# \# \#}$ \\
MI+Fosinopril & & $358.72 \pm 11.05^{\# \#}$ & $267.88 \pm 8.64^{\#}$ & $90.84 \pm 3.28^{\# \#}$ & $337.68 \pm 5.13^{\# \#}$ & $291.78 \pm 4.65^{\#}$ & $45.89 \pm 1.23^{\# \#}$ \\
\hline Sham & & $428.24 \pm 16.97$ & $327.46 \pm 14.02$ & $100.78 \pm 3.23$ & $368.68 \pm 7.45$ & $322.32 \pm 7.04$ & $46.36 \pm 0.72$ \\
MI & $4 \mathrm{wk}$ & $467.81 \pm 24.91$ & $332.99 \pm 16.64$ & $134.82 \pm 8.66^{* *}$ & $384.77 \pm 9.80$ & $324.71 \pm 7.86$ & $60.05 \pm 2.24^{* * *}$ \\
MI+XJEK & & $348.88 \pm 19.14^{\# \#}$ & $257.17 \pm 18.13^{\# \#}$ & $91.71 \pm 1.80^{\# \# \#}$ & $331.76 \pm 9.48^{\# \# \#}$ & $283.98 \pm 10.52^{\#}$ & $47.78 \pm 1.28^{\# \# \#}$ \\
MI+Fosinopril & & $415.35 \pm 17.90$ & $311.45 \pm 16.26$ & $103.90 \pm 4.08^{\# \#}$ & $362.73 \pm 7.99$ & $313.69 \pm 8.59$ & $49.04 \pm 1.99^{\# \#}$ \\
\hline Sham & & $447.41 \pm 13.95$ & $333.27 \pm 16.07$ & $113.43 \pm 4.43$ & $376.25 \pm 5.61$ & $323.73 \pm 7.51$ & $51.81 \pm 2.56$ \\
MI & $6 \mathrm{wk}$ & $570.04 \pm 41.35^{*}$ & $421.32 \pm 24.98^{*}$ & $148.72 \pm 17.33$ & $423.50 \pm 15.27^{*}$ & $364.79 \pm 10.72^{*}$ & $58.70 \pm 5.08$ \\
MI+XJEK & & $457.76 \pm 17.77$ & $341.10 \pm 19.27$ & $116.66 \pm 4.35^{\#}$ & $381.28 \pm 7.44$ & $328.70 \pm 9.42$ & $52.58 \pm 2.71^{\#}$ \\
MI+Fosinopril & & $409.38 \pm 23.97^{\#}$ & $303.47 \pm 19.51^{\#}$ & $105.91 \pm 6.26^{\# \#}$ & $359.23 \pm 10.63^{\#}$ & $308.87 \pm 10.37$ & $50.37 \pm 2.30^{\# \# \#}$ \\
\hline
\end{tabular}

TAA: area of total aorta; LA: area of lumen; CSA: cross-sectional area; AR: aorta radius; LR: luminal radius; MT: media thickness. (mean \pm SEM, $n=8 \sim 10)$. Data are represented as mean \pm SEM $(n=8 \sim 10) .{ }^{*} P<0.05,{ }^{* *} P<0.01$, and ${ }^{* * *} P<0.001$ versus sham group, ${ }^{\#} P<0.05,{ }^{\# \#} P<0.01$, and ${ }^{\# \# \#} P<0.001$ versus MI group. 

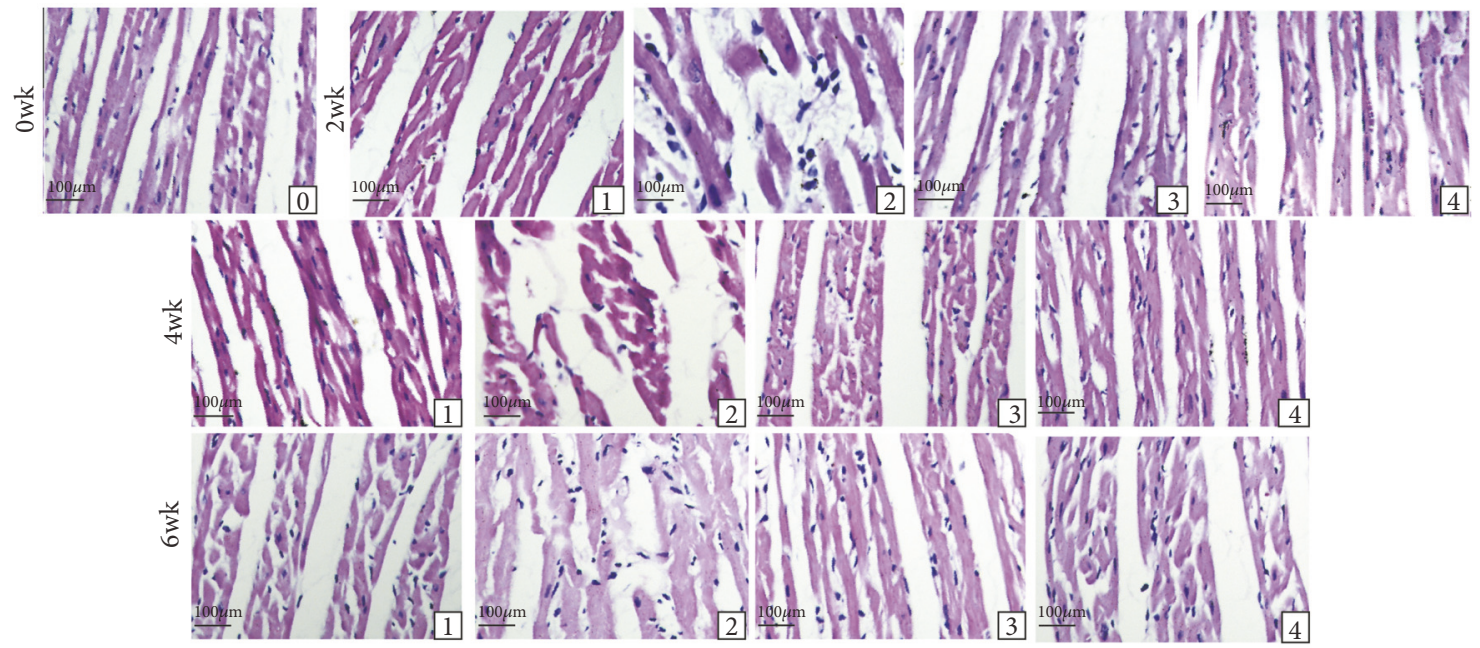

(a)

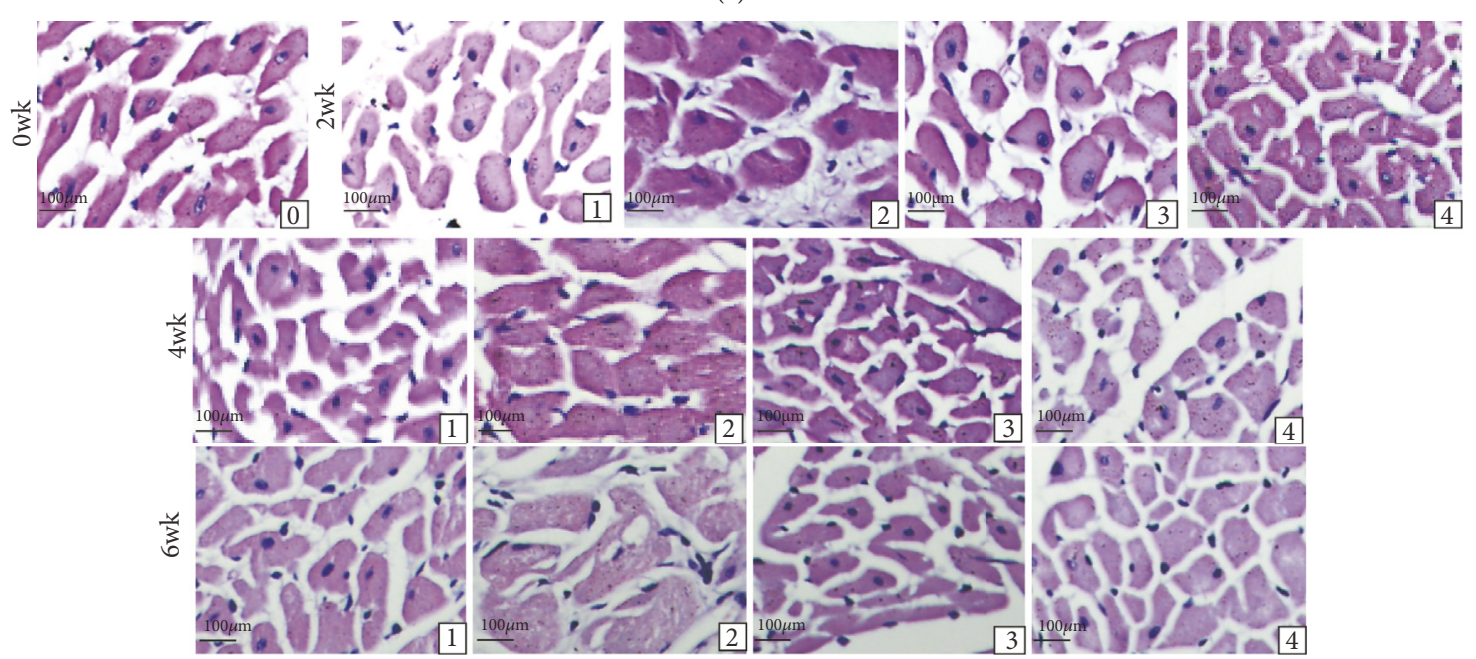

(b)

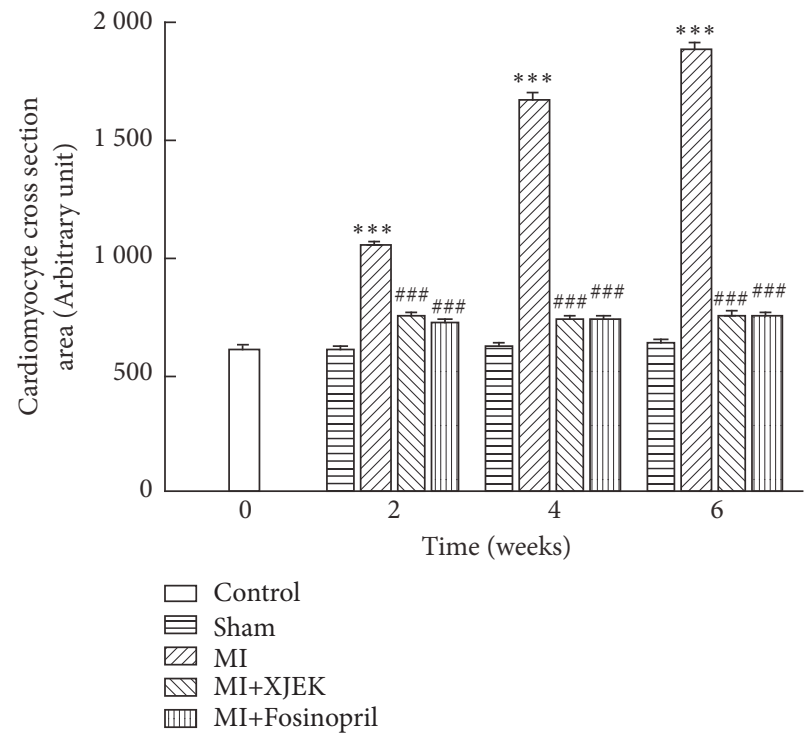

(c)

FIGURE 5: Effects of XJEK on cardiomyocyte CSA, long axis in MI rats. (HE stain, magnification×200). (a) Representative images of histological section of cardiomyocyte long axis; (b) representative images of histological section of cardiomyocyte cross-section; (c) Quantitative analyses results. (0) Control group; (1) sham group; (2) MI group; (3) MI+XJEK group; (4) MI+Fosinopril group. Data are represented as mean \pm SEM $(n=8 \sim 10) .{ }^{* * *} P<0.001$ versus sham group and ${ }^{\# \# \#} P<0.001$ versus MI group. 


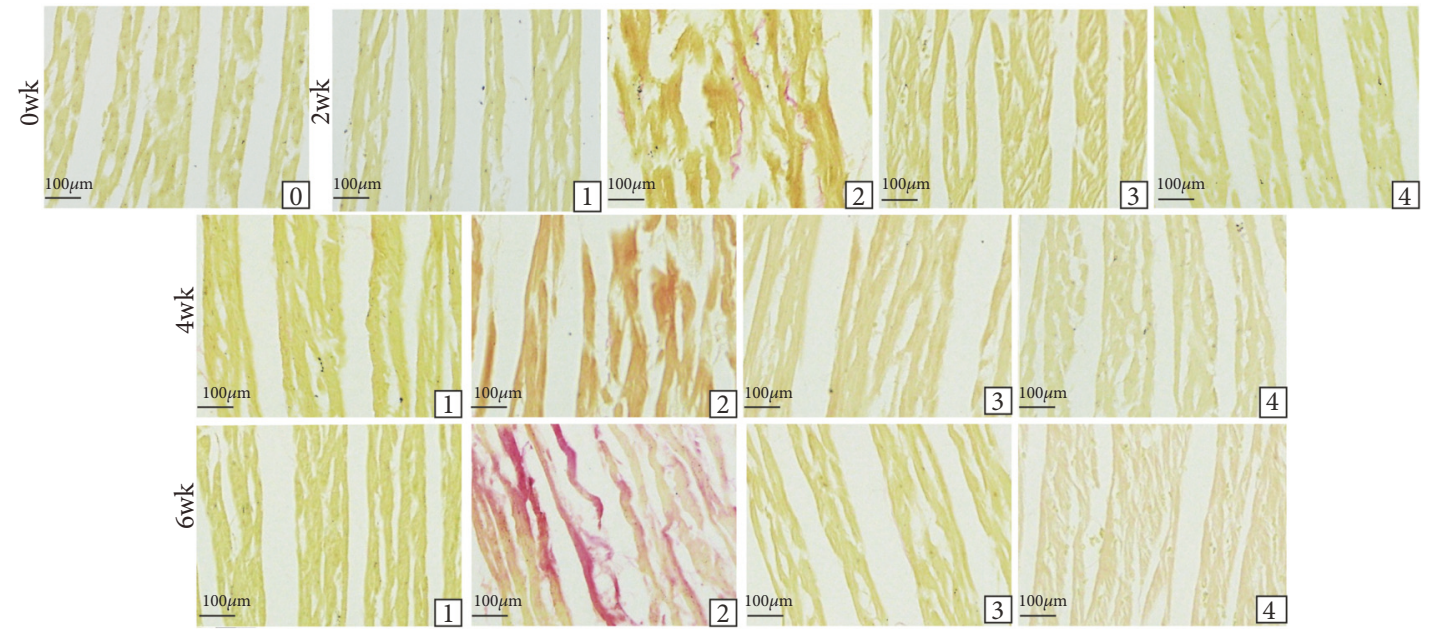

(a)
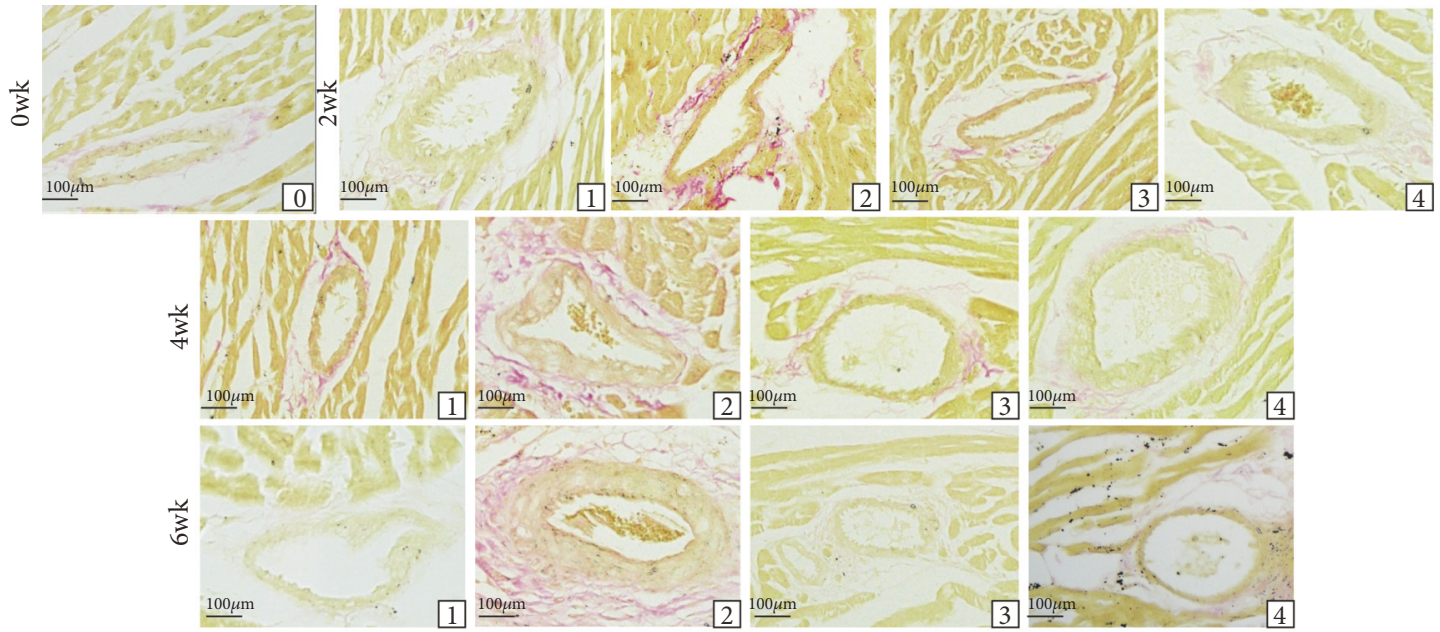

(b)

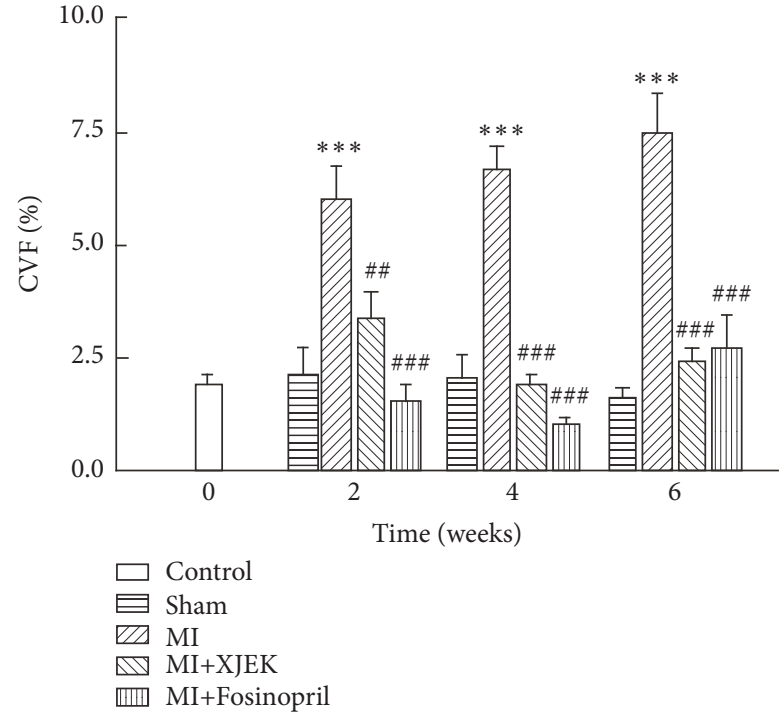

(c)



(d)

FIGURE 6: Effects of XJEK on cardiac tissue CVF and PVCA in MI rats. (a) Representative images of histological section of CVF; (b) Representative images of histological section of PVCA. ((c) and (d)) Quantitative analyses results. (0) Control group; (1) sham group; (2) MI group; (3) MI+XJEK group; (4) MI+Fosinopril group. Data are represented as mean \pm SEM $(n=8 \sim 10) .{ }^{* *} P<0.01$ and ${ }^{* * *} P<0.001$ versus sham group, ${ }^{\# \#} P<0.01$ and ${ }^{\# \# \#} P<0.001$ versus MI group. 


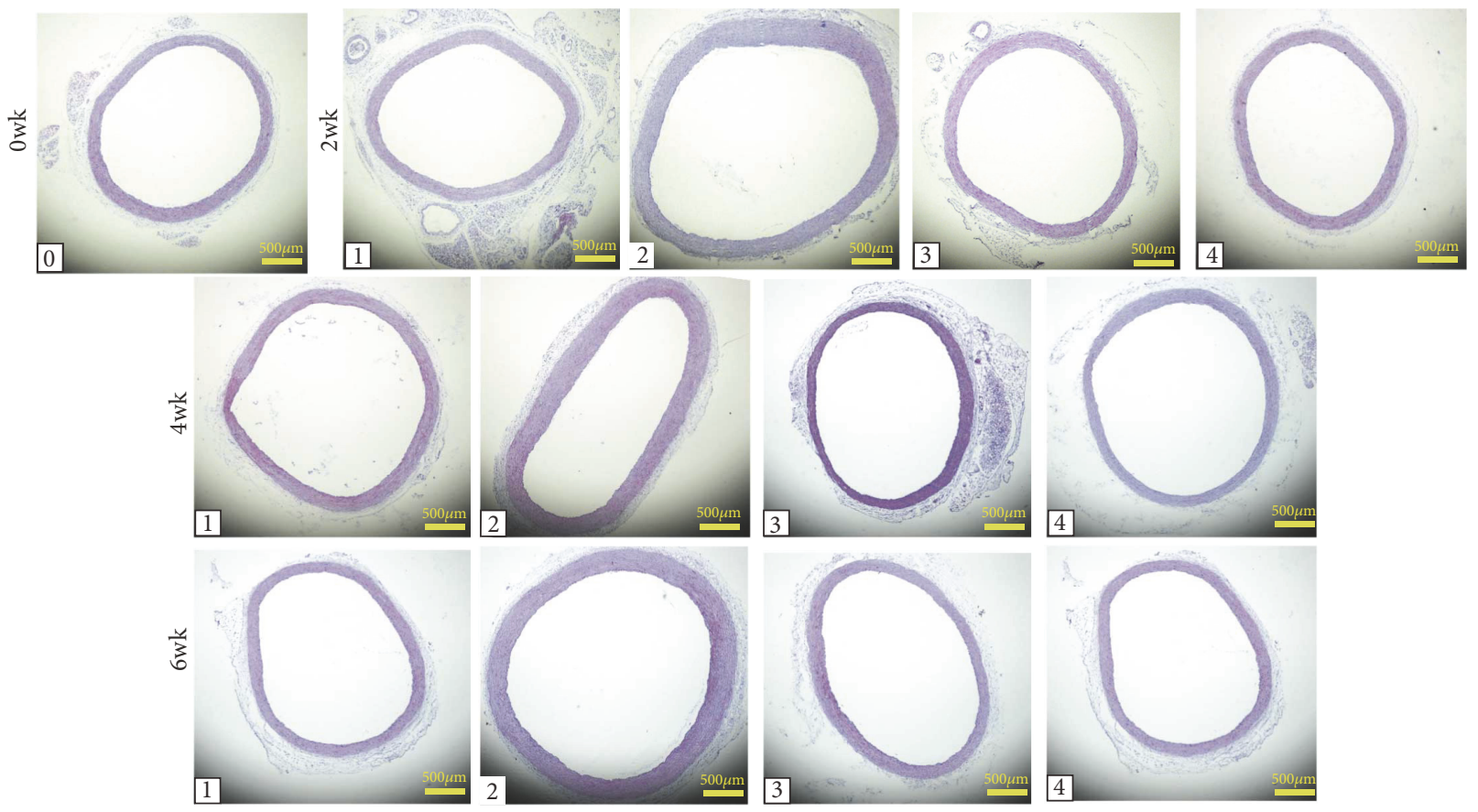

FIGURE 7: Effects of XJEK on thoracic aorta remodeling in MI rats. (HE stain, magnification $\times 40)$. (0) Control group; (1) sham group; (2) MI group; (3) MI+XJEK group; (4) MI+Fosinopril group.

groups, serum ADMA levels tended to rise in MI groups for 2, 4, and $6 \mathrm{wk}$ ( $4 \mathrm{wk}$ and $6 \mathrm{wk}, P<0.05$.), while evidently descended in XJEK ( $2 \mathrm{wk}, P<0.05$ and $6 \mathrm{wk}, P<0.01)$ and Fosinopril (6wk, $P<0.05)$ treatment groups for 2,4 , and $6 \mathrm{wk}$ (Figure 8(d)).

\subsection{Effects of XJEK on ET-1 and Ang II Content in Serum} and Cardiac Tissues of MI Rats. ET-1 levels in serum and cardiac tissues of rats in MI groups for 2, 4, and 6 wk were examined by ELISA. Compared with the corresponding sham groups, the ET-1 levels in serum $(2 \mathrm{wk}, 6 \mathrm{wk} P<0.01$ and 4 wk $P<0.05)$ and cardiac tissues $(2,4$, and 6 wk $P<0.05)$ were upregulated continuously in MI groups for 2,4 , and 6 wk (Figures 9(a) and 9(b)). However, the levels of ET-1 were significantly downregulated by XJEK or Fosinopril treatment for 2, 4, and 6wk (Figures 9(a) and 9(b)).

Similarly, Ang II levels in serum and cardiac tissues of rats in MI groups for 2, 4, and 6wk were determined. In comparison with sham groups, Ang II levels in serum (2, 4 , and 6wk $P<0.05$ ) and cardiac tissues (4wk $P<0.01$ and 6 wk $P<0.05$ ) increased evidently over time (Figures $9(\mathrm{c})$ and 9(d)). XJEK or Fosinopril treatment groups for 2, 4, and 6wk resulted in a marked decline in serum and cardiac tissue Ang II levels compared with MI groups (Figures 9(c) and 9(d)).

3.9. Effects of XJEK on $E T_{A}$ Content in Cardiac Tissues of MI Rats. The expression of $\mathrm{ET}_{\mathrm{A}}$ in cardiac tissues was examined by immunofluorescence staining, ELISA, and Western blot. There was virtually few stainings in sham groups, but in MI groups for 2, 4, and $6 \mathrm{wk}$, intense green fluorescence indicated a higher expression level of $\mathrm{ET}_{\mathrm{A}}$ compared with sham groups.
XJEK or Fosinopril treatment for 2, 4, and 6wk obviously inhibited the increased $\mathrm{ET}_{\mathrm{A}}$ levels (Figure 10(a)).

As shown in Figure 10(b) by ELISA detection, ET $_{\mathrm{A}}$ protein expression in cardiac tissues of MI rats was significantly higher than that in sham groups ( $4 \mathrm{wk}, P<0.05$ and $6 \mathrm{wk}$, $P<0.01)$, but was evidently reduced in XJEK $(2,4$ and $6 \mathrm{wk}$, $P<0.05$ ) or Fosinopril (2wk and $4 \mathrm{wk}, P<0.05$, 6wk, $P<0.01$ ) treatment for 2, 4, and 6wk (Figure 10(b)).

Consistently, the $\mathrm{ET}_{\mathrm{A}}$ content in cardiac tissues of $\mathrm{MI}$ rats also had an obvious tendency to increase (2wk and $4 \mathrm{wk}$, $P<0.01$, 6wk, $P<0.05)$, compared with sham rats. However, both XJEK ( $2 \mathrm{wk}$ and $4 \mathrm{wk}, P<0.01,6 \mathrm{wk}, P<0.05$ ) and Fosinopril $(2,4$, and $6 \mathrm{wk}, P<0.05)$ treatment could suppress the $\mathrm{ET}_{\mathrm{A}}$ expression significantly (Figures 10(c), 10(d), and 10(e)).

3.10. Effects of XJEK on eNOS Content in Serum and Cardiac Tissues of MI Rats. The expression of eNOS in the serum and cardiac tissues was examined by ELISA. The result showed that there were no significant differences in eNOS expression among all the experimental groups (Figures $11(\mathrm{~g})$ and $11(\mathrm{~h})$ ).

To provide insights into the mechanisms underlying the observed protective effects of XJEK treatment on endothelial function, we probed into the effect of XJEK on the expression of total eNOS and eNOS dimer/ (dimer+monomer) ratio in cardiac tissues by Western bolt. There was no significant difference in total eNOS expression among sham, MI, XJEK, and Fosinopril treatment for 2, 4, and 6wk. On the other hand, the eNOS dimer/ (dimer+monomer) ratio was apparently lower in cardiac tissues of MI rats for 2, 4 and $6 \mathrm{wk}$ (2wk and 6wk, $P<0.05$, $4 \mathrm{wk}, P<0.01$ ). But XJEK treatment significantly increased the dimer/ (dimer+monomer) ratio of eNOS protein expression (2wk, $P<0.05$, $4 \mathrm{wk}$ and $6 \mathrm{wk}$, 

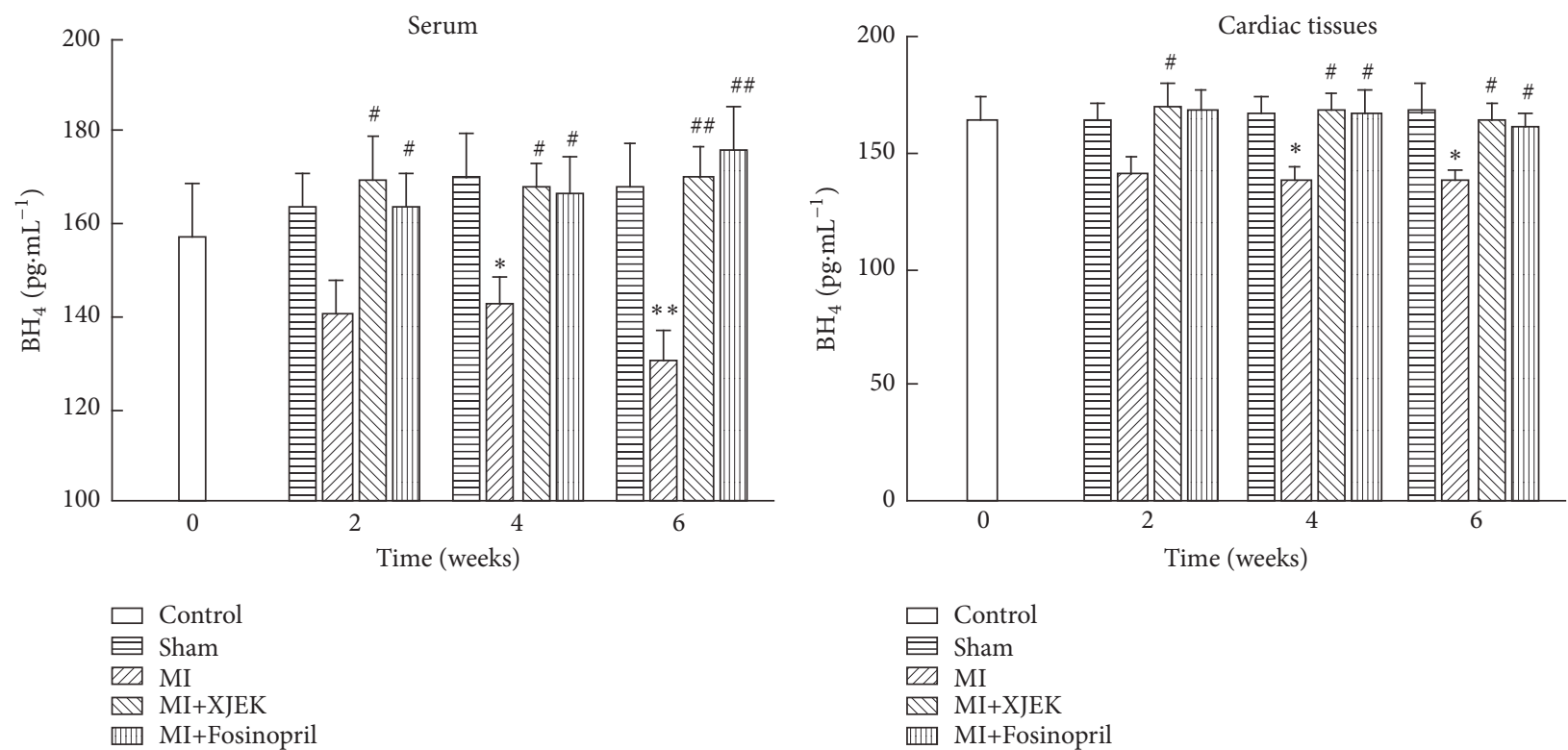

$\square$ Control

曰 Sham

VZA MI

DV MI+XJEK

血 MI+Fosinopril

(a)

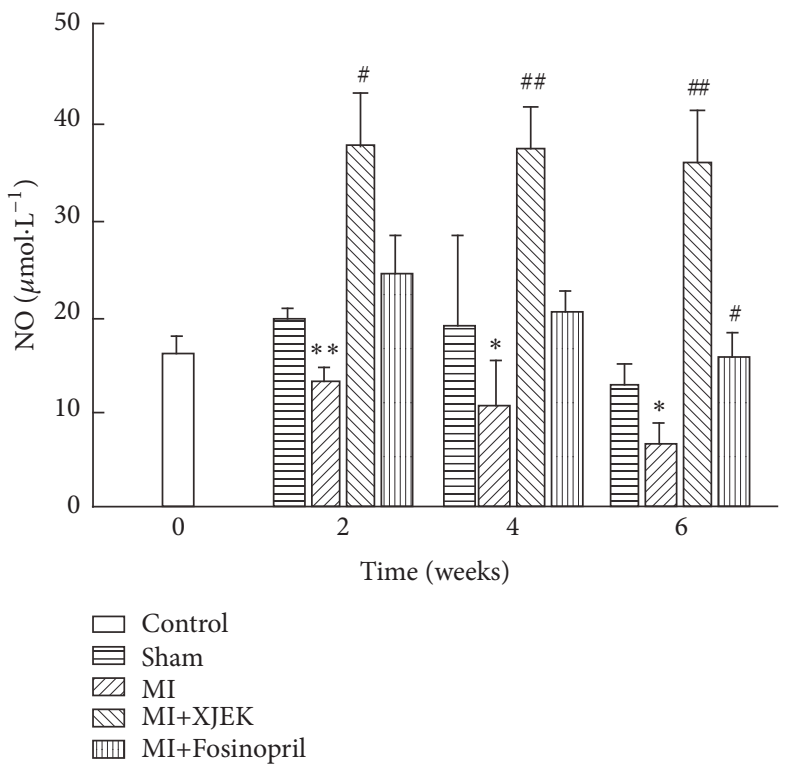

(c)

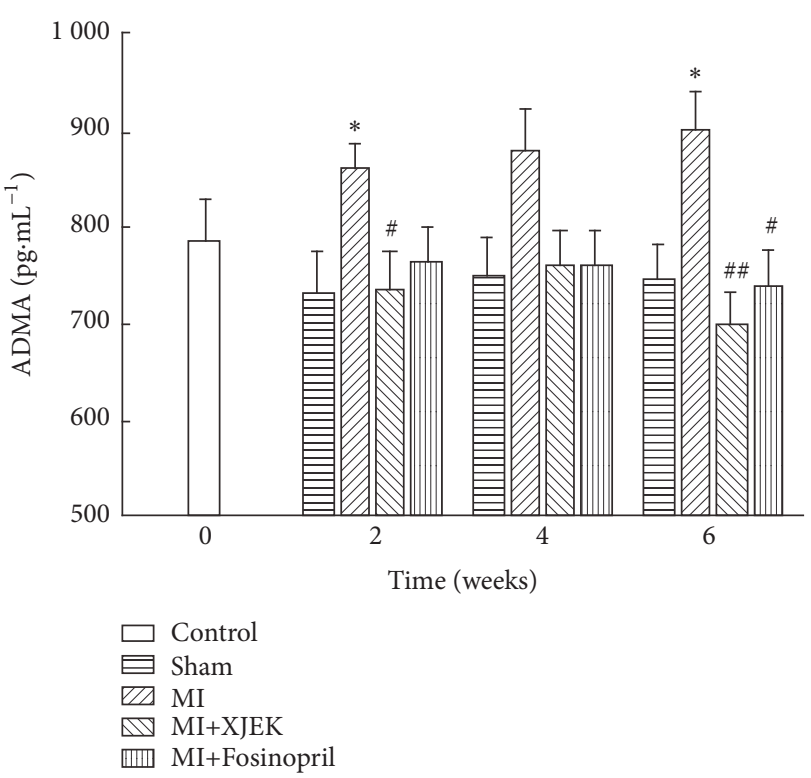

(d)

FIgURE 8: Effects of XJEK on $\mathrm{BH}_{4}$, NO and ADMA content in serum of $\mathrm{MI}$ rats. (a) $\mathrm{BH}_{4}$ content in serum; (b) $\mathrm{BH}_{4}$ content in cardiac tissues; (c) NO content in serum; (d) ADMA content in serum. Data are represented as mean \pm SEM $(n=8 \sim 10) .{ }^{*} P<0.05$ and ${ }^{* *} P<0.01$ versus sham group, ${ }^{\#} P<0.05$, and ${ }^{\# \#} P<0.01$ versus MI group.

$P<0.01)$. Fosinopril also markedly ameliorated the reduced eNOS dimer/ (dimer+monomer) ratio similarly to that by XJEK treatment $(2,4$, and $6 \mathrm{wk}, P<0.01$; Figures $11(\mathrm{~d}), 11(\mathrm{e})$, and $11(\mathrm{f}))$.

Altogether, these findings have indicated that MI is associated with the progression of eNOS uncoupling, but not total eNOS expression, in cardiac tissues of MI rats. XJEK could inhibit the eNOS uncoupling and thereby protecting endothelial function.

\section{Discussion}

In the present study, we found that XJEK significantly (1) suppressed the cardiovascular remodeling and ECG remodeling and improved cardiac function abnormalities in a rat model of MI for 2, 4 and 6wk; (2) alleviated the increasing levels of ET-1, $\mathrm{ET}_{\mathrm{A}}$ and Ang II of each time point; (3) inhibited the reduction of $\mathrm{NO}, \mathrm{BH}_{4}$ content and eNOS dimer/ (dimer+monomer) ratio therefore ameliorated ED. These 

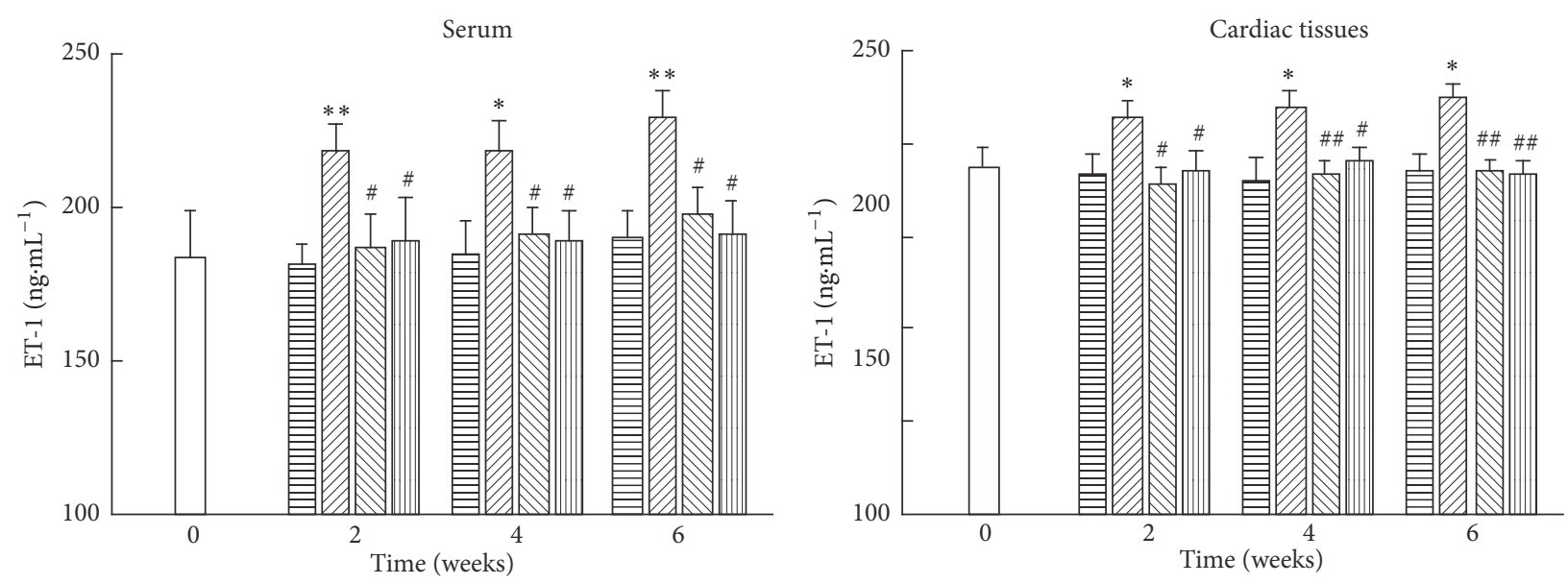

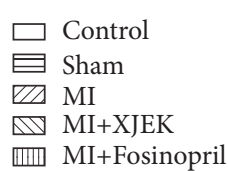

(a)

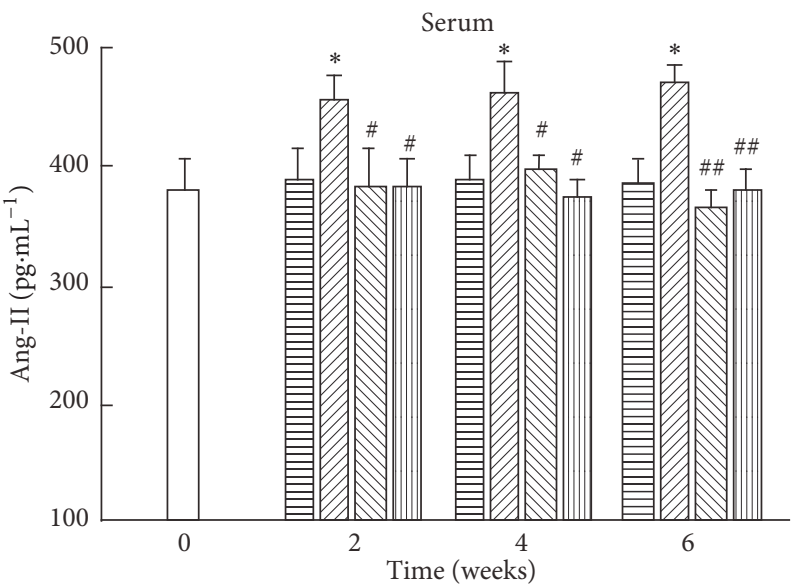

$$
\begin{aligned}
& \square \text { Control } \\
& \text { Е Sham } \\
& \text { EZA MI } \\
& \text { MI MI+XJEK } \\
& \text { 血ll MI+Fosinopril }
\end{aligned}
$$

(c)

$$
\begin{aligned}
& \square \text { Control } \\
& \square \text { Sham } \\
& \square \text { MI } \\
& \text { ㅁ. MI+XJEK } \\
& \text { 血 MI+Fosinopril }
\end{aligned}
$$

(b)

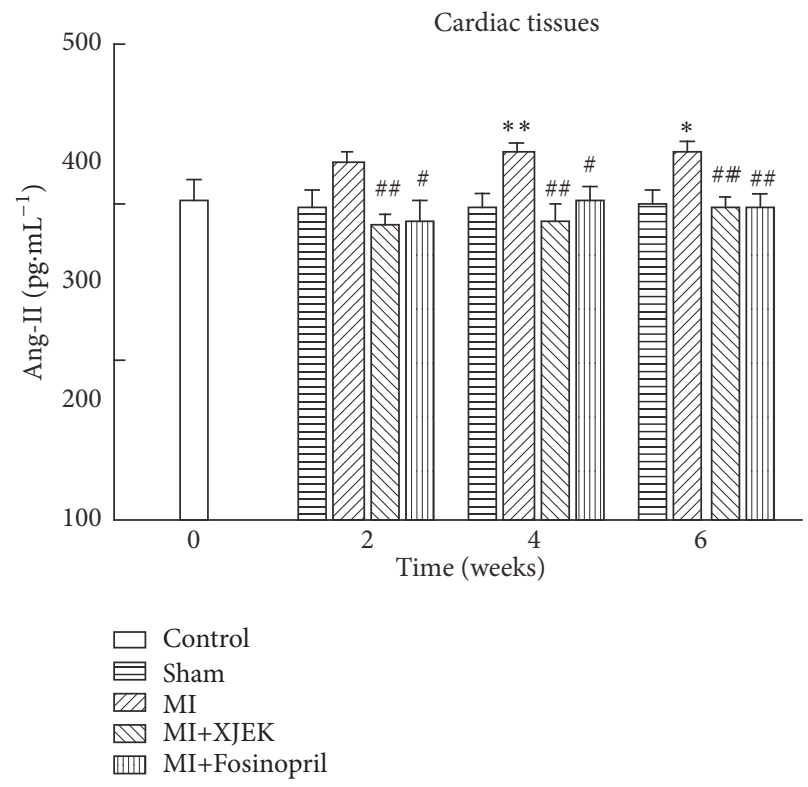

(d)

FIGURE 9: Effects of XJEK on ET-1, and Ang II content in serum and cardiac of MI rats. (a) ET-1 levels in serum; (b) ET-1 levels in cardiac tissues; (c) Ang II levels in serum; (d) Ang II levels in cardiac tissues. Data are represented as mean \pm SEM $(n=8 \sim 10)$. ${ }^{*} P<0.05$ and ${ }^{* *} P<0.01$ versus sham group, ${ }^{\#} P<0.05,{ }^{\# \#} P<0.01$, and ${ }^{\# \# \#} P<0.001$ versus MI group.

results indicate that XJEK exerts a continuously beneficial effect on MI induced by LAD ligation in rats, which may be at least in part due to the restoration of cardiovascular structures, cardiac function, and especially endothelial function.

The very surgical ligation of animal LAD could simulate the clinical situation of MI, which has been extensively used for the pathophysiological study of post-MI CR [4, 25]. Being related to alterations in geometry, size and molecular phenotype, and MI-derived CR potentiates the development of ventricular arrhythmias, left ventricle (LV) dilatation and subsequent cardiac function abnormalities [26]. Of note, excessive myocardial fibrosis in MI rats contributes to diastolic and eventually systolic dysfunction by increasing myocardial stiffness and reducing pumping capacity [27]. Herein, cardiac function in this model becomes complicated due to its association with the process of infarct expansion, cardiac hypertrophy and ventricular remodeling. Moreover, LV hypertrophy and fibrosis post-MI may result in ECG remodeling which involves prolonged action potential duration and abnormal heart beats [28]. According to a recent 

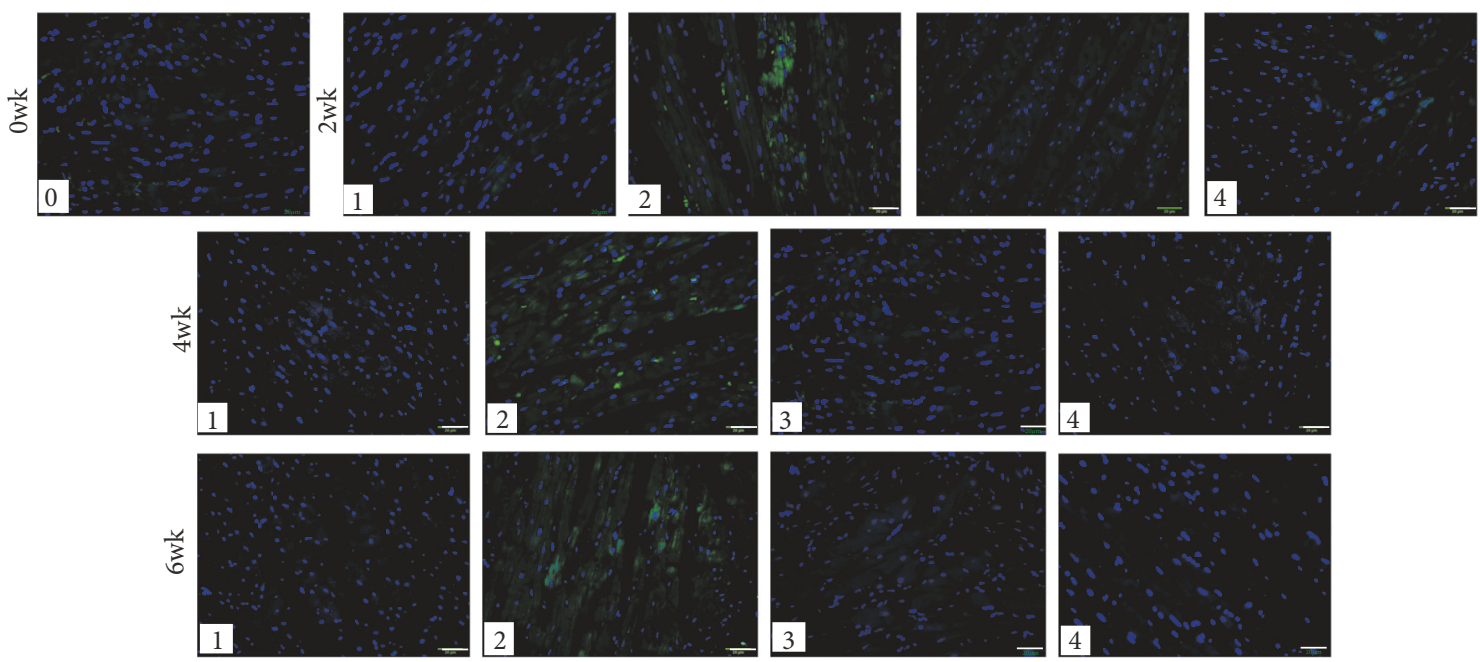

(a)
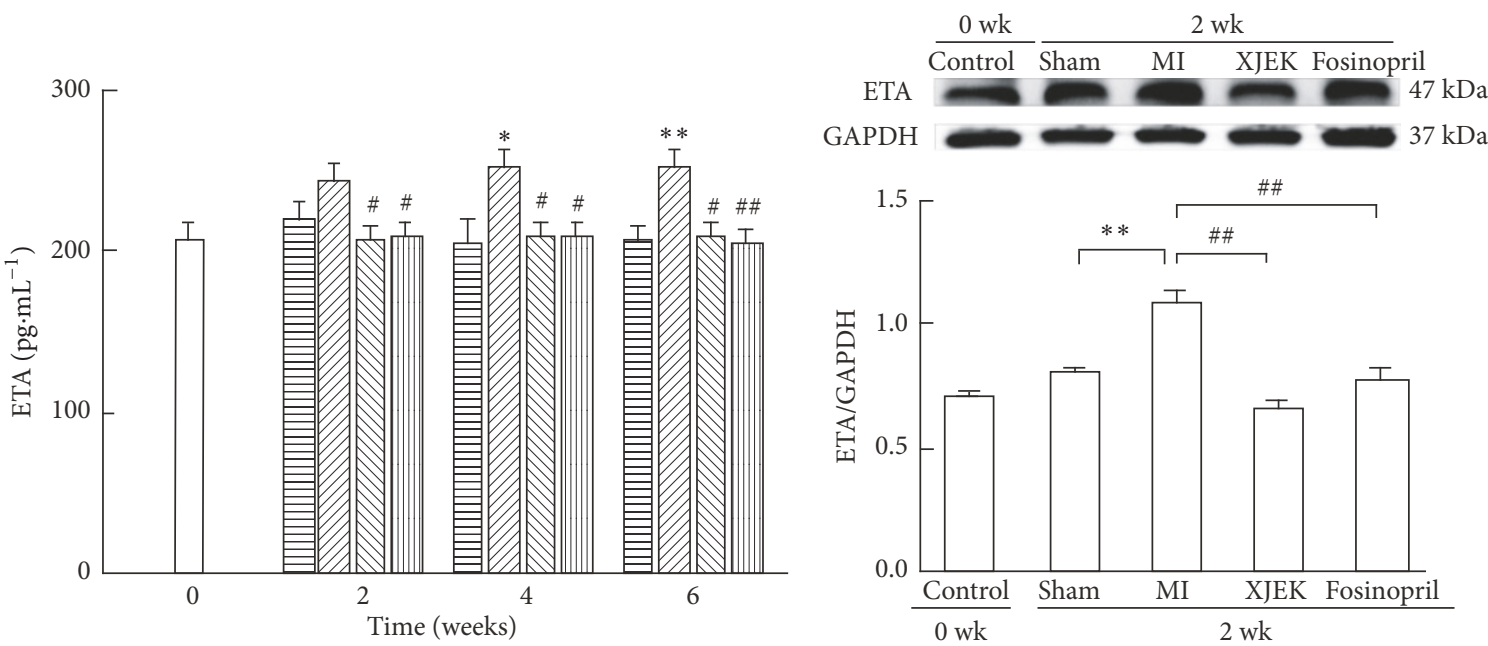

\begin{tabular}{|c|c|}
\hline$\sqsupset c$ & Control \\
\hline$\Xi \mathrm{s}$ & Sham \\
\hline $\begin{array}{l}\square \Delta 1 \\
\square \nabla\end{array}$ & M \\
\hline & \\
\hline
\end{tabular}

(b)
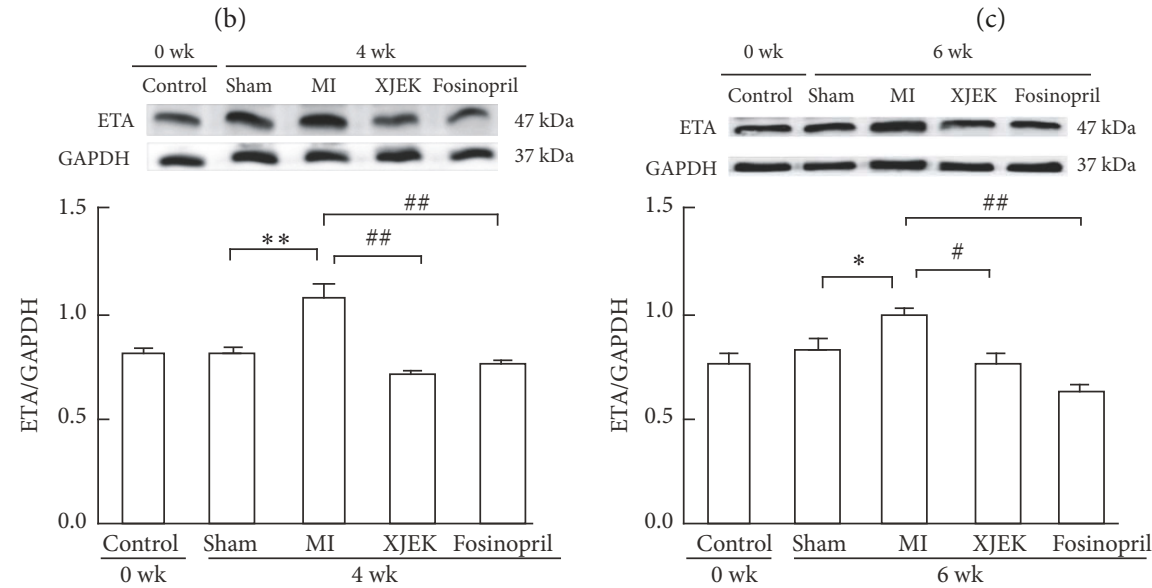

(d)

(e)

FiguRE 10: Effects of XJEK on ET $T_{A}$ expression in MI rats. (a) Representative images of Immunofluorescence of ETA (green) and DAPI (blue). (Scar bars: $20 \mu \mathrm{m}$ ); (b) $\mathrm{ET}_{\mathrm{A}}$ levels in cardiac tissues by ELISA; ((c), (d) and (e)) $\mathrm{ET}_{\mathrm{A}}$ content in cardiac tissues of rats for 2, 4 and $6 \mathrm{wk}$ by Western blot, respectively (mean \pm SEM, $n=3$ ). (0) Control group; (1) sham group; (2) MI group; (3) MI+XJEK group; (4) MI+Fosinopril group. Data are represented as mean \pm SEM $(n=8 \sim 10) .{ }^{*} P<0.05$ and ${ }^{* *} P<0.01$ versus sham group, ${ }^{\#} P<0.05$ and ${ }^{\# \#} P<0.01$ versus MI group. 


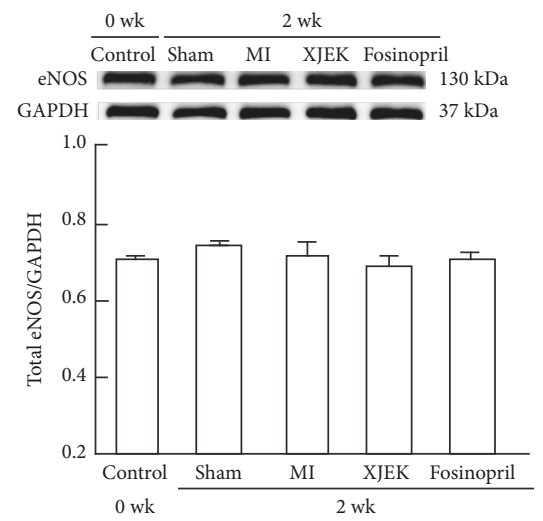

(a)
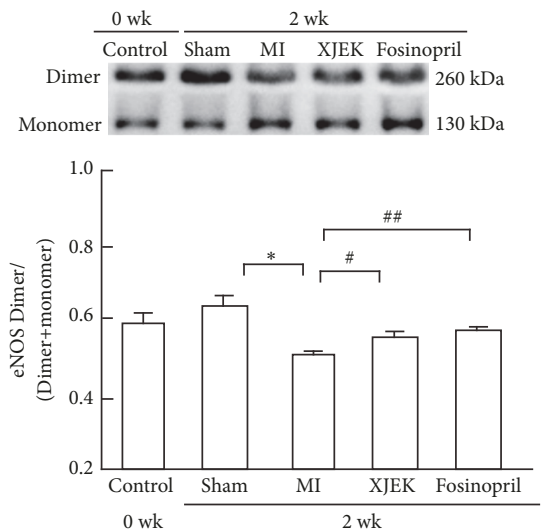

(d)

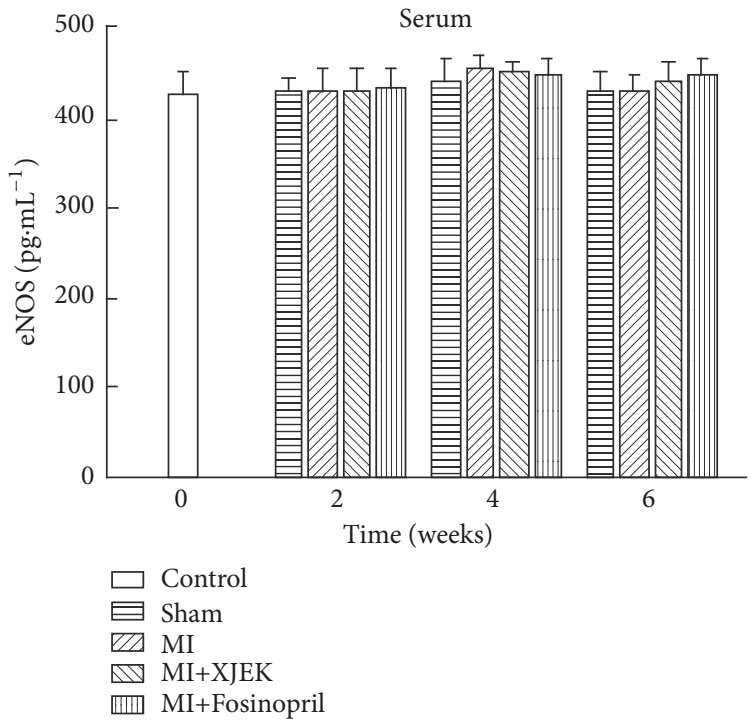

(g)

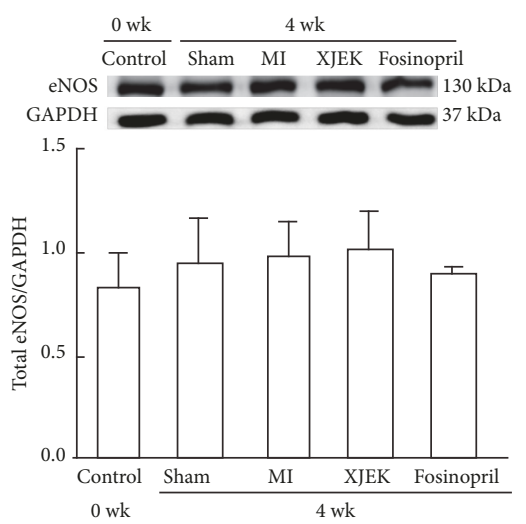

(b)

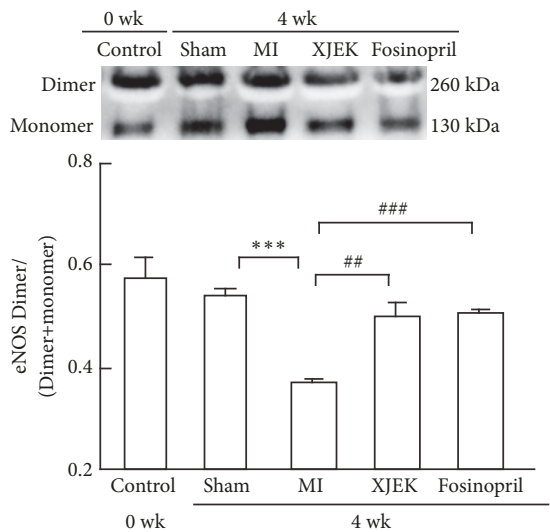

(e)

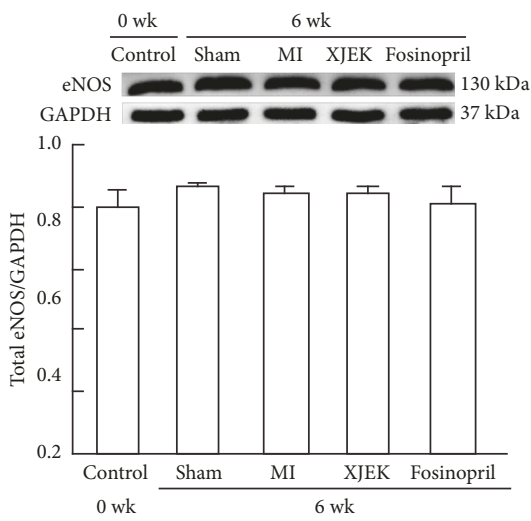

(c)

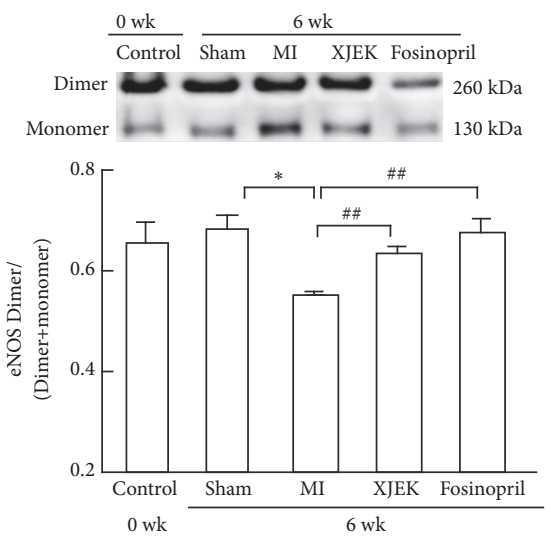

(f)

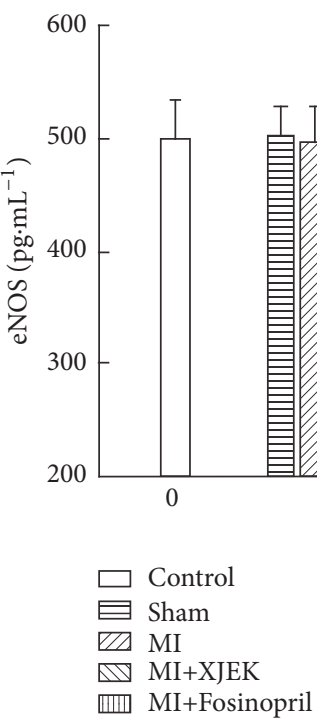

Cardiac tissues

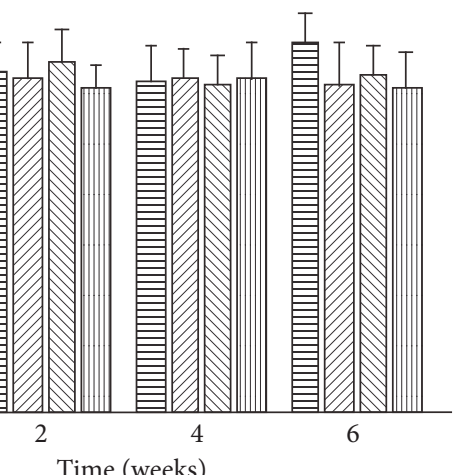

(h)

FIGURE 11: Effects of XJEK on eNOS expression in serum and cardiac tissues of MI rats. ((a), (b) and (c)) Representative Western blots of total eNOS expression in cardiac tissues and quantitative analyses of total eNOS standardized to GAPDH, data are represented as mean \pm SEM $(n=3) ;((d),(e)$ and (f)) the same protein samples as in (a), (b), or (c) were subjected to low-temperature SDS-PAGE to assess eNOS dimers and monomers in cardiac tissues; ratio of eNOS dimer/ (dimer +monomer), data are represented as mean \pm SEM ( $n=3$ ); (g) eNOS expression in serum by ELISA, data are represented as mean \pm SEM $(n=8 \sim 10)$; (h) eNOS expression in cardiac tissues by ELISA, data are represented as mean \pm SEM $(n=8 \sim 10) .{ }^{*} P<0.05$ and ${ }^{* * *} P<0.001$ versus sham group, ${ }^{\#} P<0.05,{ }^{\# \#} P<0.01$, and ${ }^{\# \# \#} P<0.001$ versus MI group. 
study, cardiac arrhythmia disruption is potential risk factor for cardiac ischemia, sudden cardiac death, and stroke [29]. In line with the previous studies, the results of our analysis showed marked CR as reflected by elevated HW/BW ratio and CSA, more collagen deposition, prolonged QT interval duration, elevated ST segment, and widened P, T, and QRS waves post-MI $(2,4$ and $6 \mathrm{wk})$ which aggravated over time. The ST segment elevation is the most sensitive marker for myocardial infarction, and it reflects myocardial necrosis and the consequent loss of cell membrane in an injuring myocardium. Interestingly, XJEK reduces QRS, QT interval, $\mathrm{P}$ wave and $\mathrm{T}$ wave width, indicating that XJEK exerts protective effects on ECG remodeling. The T wave represents the repolarization time, and a prolonged $\mathrm{T}$ wave may be due to a delay in recovery and the depleted energy level in the ischemic tissue. Normalization of the ST segment, P, T, and QRS waves by XJEK indicates sufficient perfusion all the way through the myocardial microvasculature. As far as cardiac function is concerned, impaired LVSP, $\pm \mathrm{dp} / \mathrm{dt}_{\max }$ and increased NT-ProBNP levels, which is recognized as a biomarker of heart failure [30], develop over time after MI. At the end of 2 and 4 weeks of the experiment, the rats with myocardial infarction were in a high hemodynamic state due to compensatory effects, while MI rats at $6 \mathrm{wk}$ were at the stage of transition from compensatory to decompensate and would even change into heart failure. However, the deteriorated CR was inhibited and the progressive cardiac function abnormalities were restored to normal condition after treatment with XJEK for 2, 4, and $6 \mathrm{wk}$.

The widely distributed vascular endothelium plays a very important role in modulating vascular tension by producing and releasing multiple endothelium-derived relaxing factors, including NO, prostacyclin, and endothelium-derived hyperpolarizing factors [31]. The evaluation of endothelial function in patients has gained increasing attention in the clinical settings due to its role as an excellent surrogate marker of cardiovascular events. Caused by the loss of $\mathrm{NO}$ and excessive vasoconstriction factors, ED has been shown to be associated with increased occurrence of MI and constitutes one of the earliest prognostic markers of cardiovascular disease [32]. Previous studies demonstrate that adequate level of endothelial NO is important for preserving normal vascular physiology, whereas decreased bioavailability of $\mathrm{NO}$ has been proposed as one of the central factors common for vascular remodeling, hypertension, and atherosclerosis [33]. eNOS is the key enzyme for NO synthesis which is expressed in the vascular endothelium widely. In common circumstance, functional eNOS works as a dimeric protein (coupled) together with the redox-sensitive cofactor $\mathrm{BH}_{4}$ and the substrate $L$-Arginine, to produce NO. In the presence of a highly oxidizing environment, exogenous $\mathrm{BH}_{4}$ is oxidized to $\mathrm{BH}_{2}$, which lacks of eNOS cofactor activity. A previous study suggested that the deletion of $\mathrm{BH}_{4}$ has been linked to eNOS uncoupling, and supplementation of $\mathrm{BH}_{4}$ is generally able to restore eNOS-mediated NO formation [34]. Uncoupled eNOS in dysfunctional endothelium generates $\mathrm{O}_{2}{ }^{-}$and $\mathrm{ONOO}^{-}$, resulting in oxidation of $\mathrm{BH}_{4}$. Insufficient $\mathrm{BH}_{4}$, in turn, causes further eNOS uncoupling. Thus a vicious feedback loop is formed, which increases oxidative stress and reduces NO bioavailability [35]. ADMA is a structural analogue of $L$-Arginine, which can inhibit eNOS activation and competitively inhibit the production of endogenous NO, leading to ED in experimental chronic myocardial injury [36]. ET-1, a potent vasoconstrictor and proinflammatory peptide released mostly by vascular endothelial cells, is the predominant isoform expressed in vasculature and is the most potent vasoconstrictor currently known [37]. To the best of our knowledge, ET-1 binds to $\mathrm{ET}_{\mathrm{A}}$ receptors em, leading to mitogenic reactions in cardiovascular cells ET1 and NO interplay seems to have a great relevance in the physiological regulation of vascular tone and blood pressure. The imbalance between ET-1 and NO systems may be responsible for the pathogenesis of ED following cardiac hypertrophy [38]. Ang II is another biologically active peptide of RAS. High levels of Ang II, a biologically active hormone, could increase ET-1 expression in endothelial cells [38] and promote collagen deposition [39], thereby aggravating MI.

Consistent with the above statements, the present study showed that rats in MI groups for 2, 4, and 6wk displayed ED, elucidated by decreased $\mathrm{NO}$ and $\mathrm{BH}_{4}$ levels, and increased ADMA content in serum, as well as excessive concentration of potent vasoconstrictors such as Ang II and ET-1. In addition to these characteristics, MI rats in our study did not show an insufficiency in total eNOS levels. In contrast, a significant decrease was observed in the eNOS dimer/ (dimer+monomer) ratio in cardiac tissues of MI rats (Figures 11(a)-11(h)), which could be partially explained by the depletion of $\mathrm{BH}_{4}$ and increased ADMA. Taken together, both increased ADMA levels and the deficiency of $\mathrm{BH}_{4}$ could impair eNOS activity, resulting in eNOS monomerization and blocking the production of $\mathrm{NO}$ and simultaneously aggravating MI. Interestingly, our observations revealed that these abnormal changes of endothelial-related factors were alleviated by XJEK treatment, pointing to the ED-protective properties of XJEK. However, this study is restricted to in vivo situations and did not clearly dissect the cellular and molecular mechanism of Xin-Ji-Er-Kang-induced cardioprotection. Therefore, further studies should be performed in vivo and in vitro to elucidate the beneficial effects of XJEK on myocardial infarction.

\section{Conclusion}

The present study confirms that XJEK continuously protects against $\mathrm{MI}$ induced cardiac injury by reversing $\mathrm{CR}$ and cardiac function abnormalities over time. More importantly, it highlights the key role of XJEK in inhibiting ED through attenuation of eNOS uncoupling following MI. Our results suggest that XJEK may be a candidate for the development of a new therapeutic drug in the treatment of MI.

\section{Abbreviations}

XJEK: Xin-Ji-Er-Kang

MI: Myocardial infarction

CR: Cardiovascular remodeling

ED: Endothelial dysfunction

TTC: Triphenyltetrazolium chloride 


\begin{tabular}{|c|c|}
\hline NO: & Nitric oxide \\
\hline ELISA: & $\begin{array}{l}\text { Enzyme-linked } \\
\text { immunosorbent assay }\end{array}$ \\
\hline ECG: & Electrocardiogram \\
\hline JT-ProBNP: & $\begin{array}{l}\mathrm{N} \text {-terminal probrain } \\
\text { natriuretic peptide }\end{array}$ \\
\hline ET-1: & Endothelin-1 \\
\hline $\mathrm{ET}_{\mathrm{A}}$ : & Endothelin receptor A \\
\hline Ang II: & Angiotensin II \\
\hline ADMA: & $\begin{array}{l}\text { Asymmetric } \\
\text { dimethylarginine }\end{array}$ \\
\hline $\mathrm{BH}_{4}$ : & Tetrahydrobiopterin \\
\hline eNOS: & Endothelial NO synthase \\
\hline ASBP: & $\begin{array}{l}\text { Artery systolic blood } \\
\text { pressure }\end{array}$ \\
\hline LVSP: & Left ventricular systolic \\
\hline LVEDP: & $\begin{array}{l}\text { Left ventricular } \\
\text { end-diastolic pressures }\end{array}$ \\
\hline$\pm \mathrm{dp} / \mathrm{dtmax}:$ & $\begin{array}{l}\text { Rate of rise of left } \\
\text { ventricular pressure }\end{array}$ \\
\hline RAS: & Renin-angiotensin system \\
\hline HE: & Hematoxylin and eosin \\
\hline VG: & Van Gieson \\
\hline CSA: & Cross-sectional area \\
\hline TAA: & Total aorta area \\
\hline AR: & Aorta radius \\
\hline MT: & Media thickness \\
\hline PVCA: & Perivascular collagen area \\
\hline CVF: & Collagen volume fraction. \\
\hline
\end{tabular}

\section{Data Availability}

The data used to support the findings of this study are available from the corresponding author upon request.

\section{Conflicts of Interest}

No conflicts of interest were asserted by the authors.

\section{Authors' Contributions}

Shan Gao and Ai-zong Shen, Ph.D., conceived and designed the experiments; Pan Cheng, Feng-zhen Lian, and Xiaoyun Wang performed the animal experiments; Pan Cheng performed the data analyses and wrote the manuscript; Guowei Cai, Guang-yao Huang, and Men-ling Chen performed the analysis with constructive discussions; all the writers read and assented the final MS. Pan Cheng, Feng-zhen Lian, and Xiao-yun Wang contributed equally to this work.

\section{Acknowledgments}

This work was funded by National Natural Science Foundation of China (nos. 81873126, 81373774), Anhui Medical University Foundation for Middle-Aged and Young Scientist Leaders of Disciplines in Science (no. 201324), the Creation of Major New Drugs in the Ministry of Science and Technology (no. 2017ZX09301012), Key Young and Middle-Aged Talents in Colleges and Universities (no. gxfxZD2016037), and Scientific Research Foundation of the Institute for Translational Medicine of Anhui Province (no. 2017zhyx40).

\section{References}

[1] G. W. Reed, J. E. Rossi, and C. P. Cannon, "Acute myocardial infarction," The Lancet, vol. 389, no. 10065, pp. 197-210, 2017.

[2] M. Bally, N. Dendukuri, B. Rich et al., "Risk of acute myocardial infarction with NSAIDs in real world use: bayesian metaanalysis of individual patient data," BMJ, vol. 357, Article ID j1909, 2017.

[3] J. T. Thackeray, H. C. Hupe, Y. Wang et al., "Myocardial inflammation predicts remodeling and neuroinflammation after myocardial infarction," Journal of the American College of Cardiology, vol. 71, no. 3, pp. 263-275, 2018.

[4] L. Xiong, Y. Liu, M. Zhou et al., "Targeted ablation of cardiac sympathetic neurons attenuates adverse postinfarction remodelling and left ventricular dysfunction," Experimental Physiology, vol. 103, no. 9, pp. 1221-1229, 2018.

[5] J. R. Klinger, S. H. Abman, and M. T. Gladwin, "Nitric oxide deficiency and endothelial dysfunction in pulmonary arterial hypertension," American Journal of Respiratory and Critical Care Medicine, vol. 188, no. 6, pp. 639-646, 2013.

[6] K. S. Edgar, O. M. Galvin, A. Collins, Z. S. Katusic, and D. M. McDonald, "BH4-mediated enhancement of endothelial nitric oxide synthase activity reduces hyperoxia-induced endothelial damage and preserves vascular integrity in the neonate," Investigative Ophthalmology \& Visual Science, vol. 58, no. 1, pp. 230241, 2017.

[7] T. Hashimoto, V. Sivakumaran, R. Carnicer et al., "Tetrahydrobiopterin protects against hypertrophic heart disease independent of myocardial nitric oxide synthase coupling," Journal of the American Heart Association, vol. 5, no. 3, Article ID e003208, 2015.

[8] B.-Y. Kang, K. K. Park, J. M. Kleinhenz et al., "Peroxisome proliferator-activated receptor $\gamma$ and microRNA 98 in hypoxiainduced endothelin-1 signaling," American Journal of Respiratory Cell and Molecular Biology, vol. 54, no. 1, pp. 136-146, 2016.

[9] Z.-Q. Chen, L. Hong, H. Wang, and Q.-L. Yin, "Effects of tongxinluo capsule on platelet activating factor, vascular endothelial function, blood flow of thrombolysis in myocardial infarction in acute myocardial infarction patients after delayed percutaneous coronary intervention," Zhongguo Zhong Xi Yi Jie He Za Zhi, vol. 36, no. 4, pp. 415-420, 2016.

[10] N. Sharifat, G. Mohammad Zadeh, M.-A. Ghaffari et al., "Endothelin-1 (ET-1) stimulates carboxy terminal Smad2 phosphorylation in vascular endothelial cells by a mechanism dependent on ET receptors and de novo protein synthesis," Journal of Pharmacy and Pharmacology, vol. 69, no. 1, pp. 6672, 2017.

[11] P. Maneesai, S. Bunbupha, U. Kukongviriyapan et al., "Asiatic acid attenuates renin-angiotensin system activation and improves vascular function in high-carbohydrate, high-fat diet fed rats," BMC Complementary and Alternative Medicine, vol. 16, no. 1, article 123, 2016.

[12] H.-M. Zhou, M.-L. Zhong, Y.-F. Zhang, W.-Y. Cui, C.-L. Long, and H. Wang, "Natakalim improves post-infarction left ventricular remodeling by restoring the coordinated balance between endothelial function and cardiac hypertrophy," International Journal of Molecular Medicine, vol. 34, no. 5, pp. 1209-1218, 2014. 
[13] Q. M. Wang, G. L. Chen, Y. J. Wang, H. S. Wang, M. H. Gao, and Y. Z. Gong, "An experimental study on inhibitory effect of xinjierkang granules on virus myocarditis," Zhongguo Zhong Yao Za Zhi, vol. 25, pp. 293-296, 2000.

[14] J. Hu, P. Cheng, G.-Y. Huang et al., "Effects of Xin-Ji-ErKang on heart failure induced by myocardial infarction: role of inflammation, oxidative stress and endothelial dysfunction," Phytomedicine, vol. 42, pp. 245-257, 2018.

[15] K. Guo, C.-Z. Lan, T.-T. Yu et al., "Effects of Xin-Ji-Er-Kang formula on $2 \mathrm{~K} 1 \mathrm{C}$-induced hypertension and cardiovascular remodeling in rats," Journal of Ethnopharmacology, vol. 155, no. 2, pp. 1227-1235, 2014.

[16] T.-T. Yu, K. Guo, H.-C. Chen et al., "Effects of traditional Chinese medicine Xin-Ji-Er-Kang formula on 2K1C hypertensive rats: role of oxidative stress and endothelial dysfunction," $B M C$ Complementary and Alternative Medicine, vol. 13, no. 1, article 173, 2013.

[17] J. Hu, Y.-X. Zhang, L. Wang et al., "Protective effects of Xinjierkang on myocardial infarction induced cardiac injury in mice," BMC Complementary and Alternative Medicine, vol. 17, no. 1 , article 338, 2017.

[18] H. Wu, Q. Tang, J. Yang, J. Ding, M. Ye, and W. Dong, "Atorvastatin ameliorates myocardial ischemia/reperfusion injury through attenuation of endoplasmic reticulum stress-induced apoptosis," International Journal of Clinical and Experimental Medicine, vol. 7, no. 12, pp. 4915-4923, 2014.

[19] F. Mu, J. Duan, H. Bian et al., "Cardioprotective effects and mechanism of Radix Salviae miltiorrhizae and Lignum Dalbergiae odoriferae on rat myocardial ischemia/reperfusion injury," Molecular Medicine Reports, vol. 16, no. 2, pp. 1759-1770, 2017.

[20] X.-Y. Wang, G.-Y. Huang, F.-Z. Lian et al., "Protective effect of Xin-Ji-Er-Kang on cardiovascular remodeling in high-salt induced hypertensive mice: role ofoxidative stress and endothelial dysfunction," Biomedicine \& Pharmacotherapy, vol. 115, Article ID 108937, 2019.

[21] S. M. Ahmed, S. A. Abdelrahman, and A. E. Salama, "Efficacy of gold nanoparticles against isoproterenol induced acute myocardial infarction in adult male albino rats," Ultrastructural Pathology, vol. 41, no. 2, pp. 168-185, 2017.

[22] Y. Bai, X.-B. Shi, Y.-Q. Zhang, Y.-L. Wang, X.-Y. Liu, and M. Esteve-Pastor, "Differences of matrix metalloproteinase 2 expression between left and right ventricles in response to nandrolone decanoate and/or swimming training in mice," Chinese Medical Journal, vol. 131, no. 2, pp. 207-212, 2018.

[23] N. Nuwayri-Salti, C. N. Karam, W. A. Al Jaroudi et al., "Effect of type-1 diabetes mellitus on the regulation of insulin and endothelin-1 receptors in rat hearts," Canadian Journal of Physiology and Pharmacology, vol. 85, no. 2, pp. 215-224, 2007.

[24] M. Hirata, K.-I. Serizawa, K. Aizawa et al., "22-Oxacalcitriol prevents progression of endothelial dysfunction through antioxidative effects in rats with type 2 diabetes and early-stage nephropathy," Nephrology Dialysis Transplantation, vol. 28, no. 5, pp. 1166-1174, 2013.

[25] G. Hendrikx, M. Bauwens, R. Wierts, F. M. Mottaghy, and M. J. Post, "Left ventricular function measurements in a mouse myocardial infarction model: comparison between 3Dechocardiography and ECG-gated SPECT,' Nuklearmedizin, vol. 55, no. 3, pp. 115-122, 2016.

[26] X. Zhou, J. Li, J. Guo et al., "Gut-dependent microbial translocation induces inflammation and cardiovascular events after STelevation myocardial infarction," Microbiome, vol. 6, no. 1, p. 66, 2018.
[27] A. Järve, S. Mühlstedt, F. Qadri et al., "Adverse left ventricular remodeling by glycoprotein nonmetastatic melanoma protein $B$ in myocardial infarction," The FASEB Journal, vol. 31, no. 2, pp. 556-568, 2017.

[28] M. Inoue, R. Noda, S. Yamaguchi et al., "Specific factors to predict large-vessel occlusion in acute stroke patients," Journal of Stroke and Cerebrovascular Diseases, vol. 27, no. 4, pp. 886891, 2018.

[29] J. Carberry, D. Carrick, C. Haig et al., "Persistence of infarct zone T2 hyperintensity at 6 months after acute ST-segment-elevation myocardial infarction: incidence, pathophysiology, and prognostic implications," Circulation: Cardiovascular Imaging, vol. 10, no. 12, 2017.

[30] M. Hoehn, Y. Zhang, J. Xu et al., "Overexpression of protein phosphatase $2 \mathrm{~A}$ in a murine model of chronic myocardial infarction leads to increased adverse remodeling but restores the regulation of $\beta$-catenin by glycogen synthase kinase $3 \beta$," International Journal of Cardiology, vol. 183, pp. 39-46, 2015.

[31] S. Godo and H. Shimokawa, "Endothelial functions," Arteriosclerosis, Thrombosis, and Vascular Biology, vol. 37, no. 9, pp. e108-e114, 2017.

[32] S.-R. Lee, I.-H. Chae, H.-L. Kim, D.-Y. Kang, S.-H. Kim, and H.-S. Kim, "Predictors of candesartan's effect on vascular reactivity in patients with coronary artery disease," Cardiovascular Therapeutics, vol. 35, no. 5, p. e12291, 2017.

[33] M. Siragusa and I. Fleming, "The eNOS signalosome and its link to endothelial dysfunction," Pflügers Archiv - European Journal of Physiology, vol. 468, no. 7, pp. 1125-1137, 2016.

[34] T. M. Leucker, Z.-D. Ge, J. Procknow et al., "Impairment of endothelial-myocardial interaction increases the susceptibility of cardiomyocytes to ischemia/reperfusion injury," PLOS ONE, vol. 8, no. 7, Article ID e70088, 2013.

[35] S. Battault, F. Singh, S. Gayrard, J. Zoll, C. Reboul, and G. Meyer, "Endothelial function does not improve with high-intensity continuous exercise training in SHR: implications of eNOS uncoupling," Hypertension Research, vol. 39, no. 2, pp. 70-78, 2016.

[36] S. Lambden, P. Kelly, B. Ahmetaj-Shala et al., "Dimethylarginine dimethylaminohydrolase 2 regulates nitric oxide synthesis and hemodynamics and determines outcome in polymicrobial sepsis," Arteriosclerosis, Thrombosis, and Vascular Biology, vol. 35, no. 6, pp. 1382-1392, 2015.

[37] A. Dushpanova, S. Agostini, E. Ciofini et al., "Gene silencing of endothelial von Willebrand Factor attenuates angiotensin IIinduced endothelin-1 expression in porcine aortic endothelial cells," Scientific Reports, vol. 6, Article ID 30048, 2016.

[38] G. Mao, Y. Cao, B. Wang et al., "The salutary influence of forest bathing on elderly patients with chronic heart failure," International Journal of Environmental Research and Public Health, vol. 14, no. 4, p. 368, 2017.

[39] R. Bomb, M. R. Heckle, Y. Sun et al., "Myofibroblast secretome and its auto-/paracrine signaling," Expert Review of Cardiovascular Therapy, vol. 14, no. 5, pp. 591-598, 2016. 

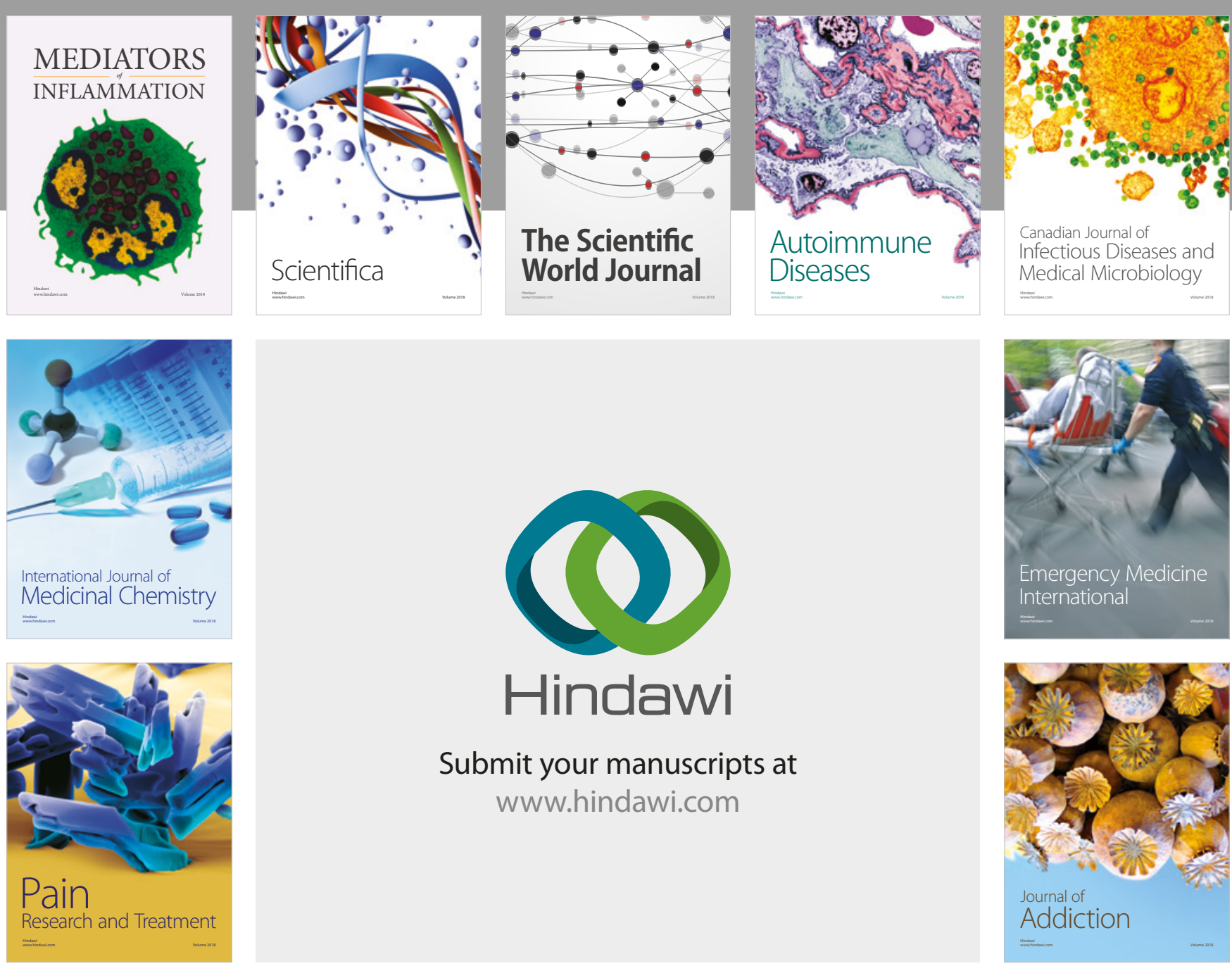

Canadian Journal of
Infectious Diseases and Medical Microbiology

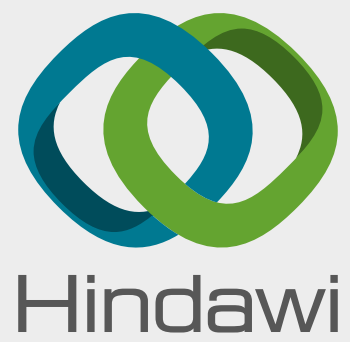

Submit your manuscripts at

www.hindawi.com
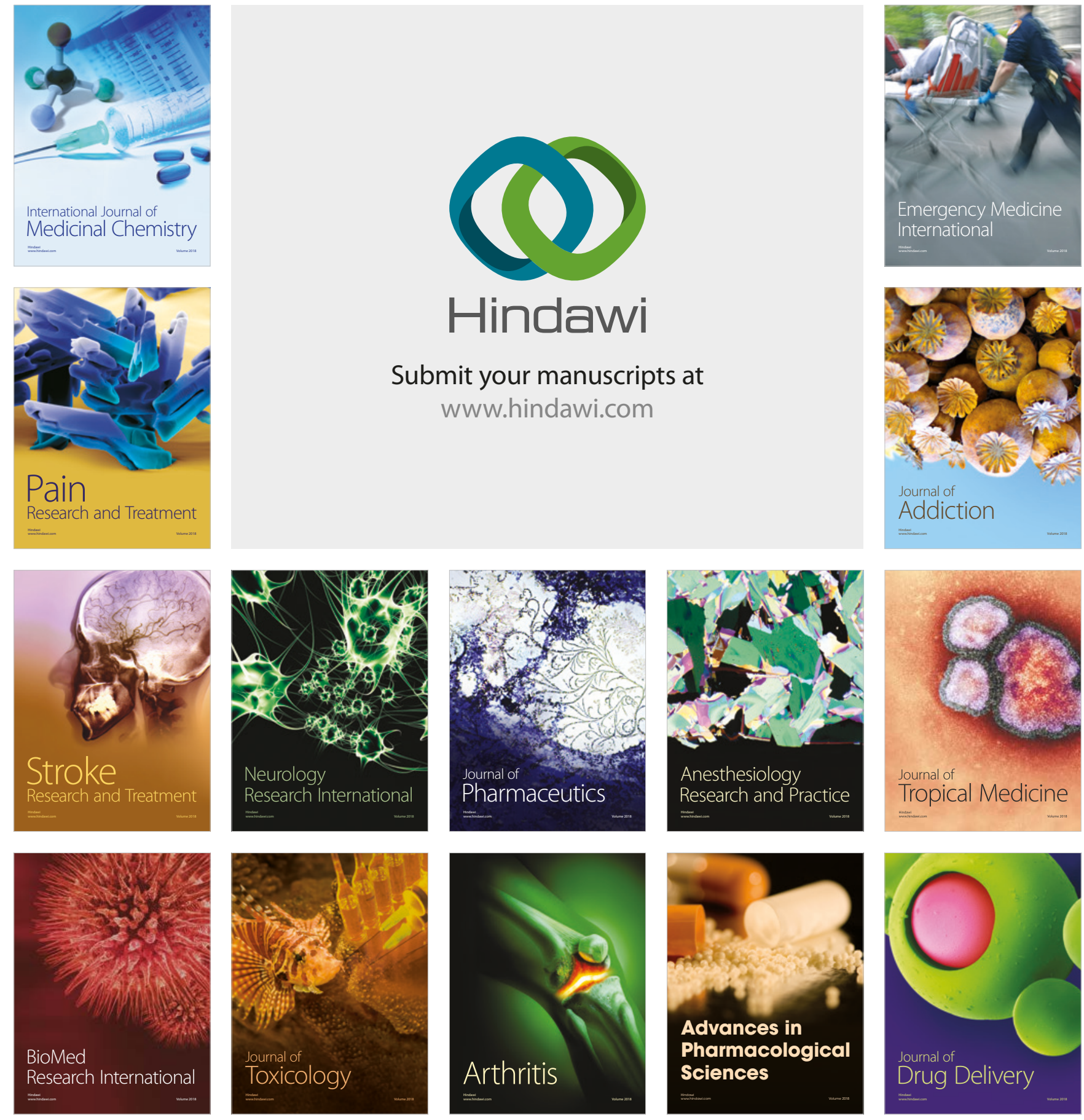